\title{
Spontaneous dimerization, critical lines, and short-range correlations in a frustrated spin-1 chain
}

\author{
Natalia Chepiga, ${ }^{1}$ Ian Affleck, ${ }^{2}$ and Frédéric Mila ${ }^{1}$ \\ ${ }^{1}$ Institute of Physics, Ecole Polytechnique Fédérale de Lausanne (EPFL), CH-1015 Lausanne, Switzerland \\ ${ }^{2}$ Department of Physics and Astronomy, University of British Columbia, Vancouver, BC, Canada V6T $1 Z 1$
}

(Received 29 August 2016; published 8 November 2016)

\begin{abstract}
We report on a detailed investigation of the spin-1 $J_{1}-J_{2}-J_{3}$ Heisenberg model, a frustrated model with nearest-neighbor coupling $J_{1}$, next-nearest neighbor coupling $J_{2}$, and a three-site interaction $J_{3}\left[\left(\mathbf{S}_{i-1} \cdot \mathbf{S}_{i}\right)\left(\mathbf{S}_{i} \cdot \mathbf{S}_{i+1}\right)+\right.$ H.c.] previously studied in [Phys. Rev. B 93, 241108(R) (2016)]. Using density matrix renormalization group (DMRG) and exact diagonalizations, we show that the phase boundaries between the Haldane phase, the next-nearest neighbor Haldane phase, and the dimerized phase can be very accurately determined by combining the information deduced from the dimerization, the ground-state energy, the entanglement spectrum and the Berry phase. By a careful investigation of the finite-size spectrum, we also show that the transition between the next-nearest neighbor Haldane phase and the dimerized phase is in the Ising universality class all along the critical line. Furthermore, we justify the conformal embedding of the SU(2) 2 Wess-Zumino-Witten conformal field theory in terms of a boson and an Ising field, and we explicitly derive a number of consequences of this embedding for the spectrum along the $\mathrm{SU}(2)_{2}$ transition line between the Haldane phase and the dimerized phase. We also show that the solitons along the first-order transition line between the Haldane phase and the dimerized phase carry a spin-1/2, while the domain walls between different dimerization domains inside the dimerized phase carry a spin 1 . Finally, we show that short-range correlations change character in the Haldane and dimerized phases through disorder and Lifshitz lines, as well as through the development of short-range dimer correlations in the Haldane phase, leading to a remarkably rich phase diagram.
\end{abstract}

DOI: 10.1103/PhysRevB.94.205112

\section{INTRODUCTION}

\section{A. Background}

Antiferromagnetic Heisenberg spin chains have been studied intensively over the years. Adding frustration through competing interactions leads to a variety of new phases and quantum phase transitions. The most famous example is probably the $J_{1}-J_{2}$ spin-1/2 chain [1] which undergoes spontaneous dimerization when the ratio of the next-nearest neighbor interaction to the nearest-neighbor one $J_{2} / J_{1} \simeq 0.2411$ [2]. For the spin-1 chain, spontaneous dimerization has long been known to be induced by a negative biquadratic interaction $J_{\text {biq }}$ exactly opposite to the bilinear one: $J_{\text {biq }} / J_{1}=-1$. The critical point is integrable with Bethe ansatz [3,4], and it is in the $\mathrm{SU}(2)_{2}$ Wess-Zumino-Witten (WZW) universality class [5-7]. Unlike in spin-1/2 chains however, a next-nearest neighbor interaction $J_{2}$ does not lead to dimerization, but induces a phase transition into a phase that consists of intertwined next nearest-neighbor (NNN) Haldane chains [8,9]. More recently, it has been shown that a three-site interaction $J_{3}\left[\left(\mathbf{S}_{i-1} \cdot \mathbf{S}_{i}\right)\left(\mathbf{S}_{i} \cdot \mathbf{S}_{i+1}\right)+\right.$ H.c. $]$ that reduces to the next-nearest neighbor interaction for spin- $1 / 2$ is also able to induce a spontaneous dimerization in spin-S chains for arbitrary $\mathrm{S}$, and that, at least up to $S=2$, the transition is in the $\mathrm{SU}(2)_{2 S} \mathrm{WZW}$ universality class $[10,11]$.

The combined effect of $J_{2}$ and $J_{\text {biq }}$ for the spin- 1 chain has recently been investigated by Pixley et al. [12], who came to the conclusion that the phase diagram only consists of the phases previously identified in the models with only one of these frustrating interactions ( $J_{2}$ or $J_{\text {biq }}$ ): the Haldane phase, a spontaneously dimerized phase, and the NNN-Haldane phase. They also carefully investigated the short-range correlations, which become incommensurate through Lifshitz and disorder transition line. The dimerization transition was argued to be either in the $\mathrm{SU}(2)_{2} \mathrm{WZW}$ universality class, or to be first order.

Shortly after, the combined effect of $J_{2}$ and of the three-site interaction $J_{3}$ has been studied by the present authors [13]. The model is defined by the $J_{1}-J_{2}-J_{3}$ Hamiltonian:

$$
\begin{aligned}
H= & \sum_{i}\left(J_{1} \mathbf{S}_{i} \cdot \mathbf{S}_{i+1}+J_{2} \mathbf{S}_{i-1} \cdot \mathbf{S}_{i+1}\right) \\
& +\sum_{i} J_{3}\left[\left(\mathbf{S}_{i-1} \cdot \mathbf{S}_{i}\right)\left(\mathbf{S}_{i} \cdot \mathbf{S}_{i+1}\right)+\text { H.c. }\right] .
\end{aligned}
$$

The phases turn out to be the same as for the $J_{1}-J_{2}-J_{\text {biq }}$ model, but, quite surprisingly, the dimerization transition between the NNN-Haldane and the dimerized phase was found to be in the Ising universality class. The goal of the present paper is to give a detailed account of how these conclusions were reached for the $J_{1}-J_{2}-J_{3}$ model, and to investigate the nature of the short-range correlations, which were not touched upon in the previous paper. The apparent discrepancy regarding the nature of the transition from the NNN-Haldane to the dimerized phase between the $J_{1}-J_{2}-J_{\text {biq }}$ and the $J_{1}-J_{2}-J_{3}$ model has been resolved since then by the present authors [14], and the transition appears to be in the Ising universality class in both cases.

\section{B. Previous results}

The spin-1 chain with isotropic nearest-neighbor Heisenberg $\left(J_{2}=J_{3}=0\right)$ interaction has been shown to be gapped [15] with exponentially decaying spin-spin correlations. The system is topologically nontrivial, and the ground state has a hidden order characterized by a nonlocal string order parameter. For open boundary conditions, spin-1/2 edge states appear and give rise to two low-lying states, a singlet and the so-called Kennedy triplet [16]. More recently, it 
was shown that the Haldane phase is characterized by the double degeneracy of the entanglement spectrum [17]. This degeneracy is protected by the same set of symmetries that protect the stability of the Haldane phase. If the Hamiltonian is deformed while preserving these symmetries, the degeneracy can be lifted only by crossing a phase boundary.

The model with $J_{3}=0$ has been studied using a variational ansatz and the density matrix renormalization group (DMRG) [8,18-20]. The authors have shown that the Haldane phase is stable until $J_{2}=0.7444(6)$, where a phase transition to the NNN-Haldane phase takes place. According to DMRG calculations, the spin gap remains open. The finite jump in the string order parameter suggests that the phase transition is first order, although no discontinuity could be identified in the first derivative of the energy at the transition. Disorder and Lifshitz points (i.e., points, where the correlation function in real space becomes incommensurate with a wave vector $q \neq 0, \pi / 2, \pi$, or where the structure factor has two peacks at $q \neq 0, \pi / 2, \pi$, respectively) were identified at $\alpha_{d}=0.284(1)$ and $\alpha_{L}=0.3725(25)$.

For the model with $J_{2}=0$, there is a transition at $J_{3} \simeq$ 0.111 [10] to a spontaneously dimerized phase. This transition is continuous and belongs to the $\mathrm{SU}(2)_{k=2} \mathrm{WZW}$ universality class [7].

There is also a line where the ground state is known exactly. Michaud et al. $[10,11]$ have shown that there is an exactly dimerized point for all spin-S chains for the $J_{1}-J_{3}$ model at $J_{3} / J_{1}=1 /(4 S(S+1)-2)$. For spin-1/2, this model reduces to the $J_{1}-J_{2}$ model with $J_{2}=J_{3} / 2$, and this exactly dimerized state can be seen as the generalization of the Majumdar-Ghosh point of the spin-1/2 $J_{1}-J_{2}$ chain. Further investigations have shown that this result can be extended to the case where a next-nearest neighbor exchange $J_{2}$ interaction is included [21]. Indeed, provided that

$$
\frac{J_{3}}{J_{1}-2 J_{2}}=\frac{1}{4 S(S+1)-2},
$$

the two fully dimerized states are eigenstates, and they are ground states if $J_{2}$ is not too large. Now, for $J_{3}=0$ and $S=1$, it has already been shown by Roth and Schollwöck that the ground state is not dimerized for $J_{2}=1 / 2$, but that it lies in the Haldane phase [8,18]. This suggests that, for spin 1, the transition between the dimerized phase and the Haldane phase, which is continuous for $J_{2}=0$, has to become first order somewhere on the line $J_{2}+3 J_{3}=1 / 2$ [the form taken by the condition of Eq. (2) for $S=1$ ].

The phase diagram of the $J_{1}-J_{2}-J_{3}$ model (Fig. 1) was reported previously in Ref. [13], in which we have discussed in details the nature of the phase transitions into the spontaneously dimerized phase. In particular, it was shown that the phase transition between the Haldane and dimerized phases is either WZW SU(2) $)_{k=2}$ or first order depending on the value of the coupling constant of the marginal operator, while the transition between the NNN-Haldane and dimerized phases is in the Ising universality class. In addition, we have suggested that the type of continuous transition depends on the nature of the domain walls between the phases: the transition is magnetic [WZW SU(2) $)_{k=2}$ ] if the domain wall carries a free spin, while it occurs in singlet sector and is in the Ising universality class otherwise.

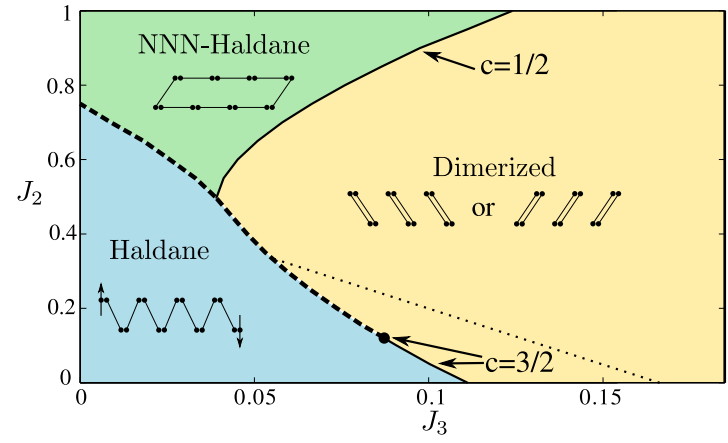

FIG. 1. Phase diagram of the one-dimensional, spin- $1 J_{1}-J_{2}-J_{3}$ model. The transition from the dimerized phase to the Haldane phase is continuous along the solid line and first order along the dashed line. The transition from the NNN-Haldane to the dimerized is always continuous. The transition between the Haldane phase and the NNNHaldane phase is always first order. The dotted line is the line where the ground state is exactly dimerized.

\section{Scope}

In this paper, we report on an in-depth numerical and analytical investigation of the model of Eq. (1) using DMRG, exact diagonalizations, and field theory. Without loss of generality, we set $J_{1}=1$ throughout the paper, and we concentrate on the antiferromagnetic case $J_{2} \geqslant 0$ and on positive three-site interaction $J_{3} \geqslant 0$. In particular, we discuss the dimerization, the ground-state energy and the entanglement spectrum, all obtained by a matrix product state implementation of DMRG known as variational MPS [22,23], and the Berry phase [24,25] calculated with exact diagonalization. We also confirm the magnetic nature of the domain walls between the Haldane and dimerized phases by looking at the solitons at the first order transition between these phases. Furthermore, coming back to the nature of the phase transition between the NNN-Haldane and dimerized phases, we provide numerical evidence that the universality class is Ising all along the critical line, including the triple point. Finally, we discuss a variety of short range phases that appear on top the main phases.

The paper is organized as follows. We start with a brief discussion of the phase diagram in Sec. II. Section III discusses the conformal embedding used in the field theory approach and provides some technical details on DMRG calculations. In Sec. IV, we describe in more details how the phase diagram was obtained by a careful investigation of the dimerization order parameter, of the energy, of the entanglement spectrum, and of two types of Berry phases. In Sec. V, we discuss solitons that appear at the first order phase transition between the Haldane and dimerized phases. Section VI discusses the limits of the Ising critical line: the triple point and the $J_{2}-J_{3}$ model. Section VII gives additional details about the short-range orders realized in the system. We conclude with a summary of our main results in Sec. VIII.

\section{SUMMARY OF MAIN RESULTS}

\section{A. Phases and transitions}

Our main results are summarized in the phase diagram of Fig. 1. It consists of three phases: a Haldane phase around 
the nearest-neighbor Heisenberg chain $\left(J_{2}=J_{3}=0\right)$, a nextnearest neighbor (NNN)-Haldane phase upon increasing $J_{2}$, and a dimerized phase upon increasing $J_{3}$. Each phase has a simple valence-bond-solid (VBS) representation sketched on the diagram. In this representation, each spin-1 is represented as a pair of spins $1 / 2$, and bonds correspond to spin singlets built out of two spins $1 / 2$.

The transition between the Haldane phase and the dimerized phase starts at $J_{2}=0$ and $J_{3} \simeq 0.111$, and it remains continuous along a line up to the point $J_{2} \simeq 0.12$ and $J_{3} \simeq 0.087$. On this line, and at the end point, the transition is characterized by a central charge $c=3 / 2$ and is in the $\mathrm{SU}(2)_{k=2} \mathrm{WZW}$ universality class. Beyond, the transition is first order.

The transition between the Haldane phase and the NNNHaldane phase is first order. It is a topological transition: the two phases cannot be distinguished by any local symmetry, but Haldane phase is topological with gapless edge excitations (so-called Kennedy triplet [16]), whereas the NNN-Haldane phase is not. One can also resort to the nonlocal string order parameter, or to probes of topological properties such as the entanglement spectrum or the Berry phase, to distinguish them.

Finally, the transition between the NNN-Haldane phase and the dimerized phase is in the Ising universality class with a central charge $c=1 / 2$. The singlet-triplet gap does not close at this transition.

On top of these transition lines, there is a remarkable line $J_{2}+3 J_{3}=1 / 2$ along which the fully dimerized state is an exact eigenstate of the model. This state is the ground state until the point $J_{2} \simeq 0.335$ and $J_{3} \simeq 0.055$. At that point, the system undergoes a strongly first order transition into the Haldane phase.

\section{B. Short-range order}

In addition to these phases, which can be distinguished by their topological properties or by the development of longrange dimerization, we have also identified regions of the phase diagrams characterized by various types of short-range order. The discussion and notations follow closely those of Ref. [12], in which a very detailed investigation of the same problem for the $J_{1}-J_{2}-J_{\text {biq }}$ model has been reported. The lack of long-range order to distinguish these regeions prevents them from being true phases in the thermodynamic sense. However, they play an important role in understanding the evolution of correlations in the phase diagram, and we will nevertheless refer to them as phases.

The correlation function $C(x)=\langle\mathbf{S}(0) \cdot \mathbf{S}(x)\rangle$ can be well accounted for throughout by the product of the twodimensional Ornstein-Zernicke (OZ) form:

$$
C_{\mathrm{OZ}}(x) \propto \cos (q x) \frac{e^{-x / \xi}}{\sqrt{x}},
$$

with, in some cases, a prefactor $1+\delta(-1)^{x}$, leading to the dimerized Ornstein-Zernicke (DOZ) form:

$$
C_{\mathrm{DOZ}} \propto\left(1+\delta(-1)^{x}\right) C_{\mathrm{OZ}}(x) .
$$

The wave number $q$, the correlation length $\xi$, and the dimerization parameter $\delta$ are fitting parameters that depend on the couplings $J_{2}$ and $J_{3}$.

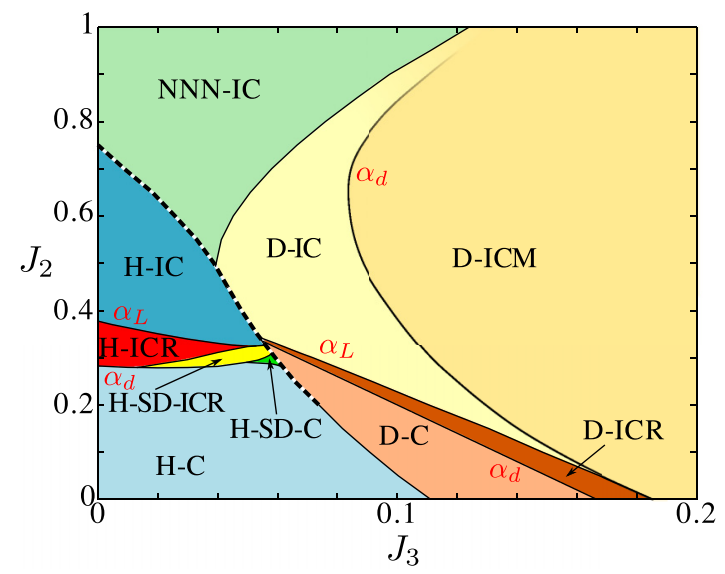

FIG. 2. Phase diagram based on the type of short-range order realized in the phases of Fig. 1. The notations for the different phases are described in the text. Note that the disorder line $\alpha_{d}$ is distinct from the Lifshitz line $\alpha_{L}$ in both Haldane and dimerized phases. The line where the fully dimerized state is an exact ground-state coincides with the disorder line $\alpha_{d}$ in the dimerized phase.

Note that the same form applies to the dimerized and nondimerized phases, except, of course, a line of continuous WZW SU(2) 2 phase transition, at which the spin-spin correlation decays algebraically $C(x) \propto(-1)^{x} /|x|^{3 / 4}$ up to logarithmic corrections. The dimerized phase is characterized by the development of long-range correlations of the two-spin operator $\mathbf{S}_{i} \cdot \mathbf{S}_{i+1}$.

The structure factor is defined by the Fourier transform of real-space correlations $\left\langle\mathbf{S}_{i} \cdot \mathbf{S}_{j}\right\rangle$ :

$$
S F(q)=\frac{1}{N} \sum_{i, j} e^{i q(i-j)}\left\langle 0\left|\mathbf{S}_{i} \cdot \mathbf{S}_{j}\right| 0\right\rangle .
$$

Various short-range commensurate and incommensurate phases are shown in Fig. 2. Below we provide a short description of each phase. The detailed discussion of the form of the correlations that led to the identification of short-range order can be found in Sec. VII.

\section{Haldane phase}

(1) H-C. Short-range antiferromagnetic order with commensurate real-space correlation function. $C(x)$ is well described by the OZ form with $q=\pi$ and no dimerization $(\delta=0)$. The structure factor $S F(q)$ has a single peak at $q=\pi$.

(2) H-SD-C. Short-range dimer phase with commensurate real-space correlations $(q=\pi) . C(x)$ is well described by the dimerized OZ form with $q=\pi$ and $\delta>0 . S F(q)$ has a single peak at $q=\pi$.

(3) H-SD-ICR. Short-range dimer phase with incommensurate real-space correlations, characterized by $q>\pi$ and $\delta>0$ in Eq. (4). $S F(q)$ has a single peak at $q=\pi$.

(4) H-ICR. Short-range antiferromagnetic order with incommensurate real-space correlations. $C(x)$ is well described by the $\mathrm{OZ}$ form with $q \neq \pi$ and no dimerization $(\delta=0)$. $S F(q)$ has a single peak at $q=\pi$.

(5) H-IC. Short-range antiferromagnetic order with incommensurate correlations in both real and momentum spaces. $C(x)$ is well described by the OZ form with $q>\pi$ and no 
dimerization $(\delta=0)$, but $S F(q)$ has two symmetric peaks at $q \neq \pi$.

\section{Dimerized phase}

(1) D-C. The spin correlations are commensurate in both, real and momentum space. $C(x)$ is well described by the dimerized DOZ form with $q=\pi$ and $\delta>0$. $S F(q)$ has a single peak at $q=\pi$.

(2) D-ICR. Real-space correlations are incommensurate, and $C(x)$ is well fitted by the DOZ form with $\delta>0$ and $q>\pi$. $S F(q)$ still has a single peak at $q=\pi$.

(3) D-IC. The spin correlations are incommensurate in both real and momentum space, $C(x)$ is well fitted by the DOZ form with $\delta>0$ and $q>\pi$, but $S F(q)$ has two symmetric peaks at $q \neq \pi$.

(4) D-ICM. The spin correlations are incommensurate in momentum space, $S F(q)$ has two symmetric peaks at $q \neq$ $\pi, \pi / 2$. Real-space correlations are commensurate with $q=$ $\pi / 2$.

\section{NNN-Haldane phase}

(1) NNN-IC. The spin-spin correlations are incommensurate in both, real and momentum space. $C(x)$ is reasonably well fitted by the OZ form with $q>\pi$ and no dimerization $(\delta=0)$. $S F(q)$ has two symmetric peaks at $q \neq \pi$.

\section{METHODS}

\section{A. Conformal embedding}

An important technique used in Ref. [13] was a conformal embedding - an exact equivalence of the $\mathrm{SU}(2)_{2} \mathrm{WZW}$ conformal field theory (CFT) with the direct product of Ising and free boson CFT's. This was convenient since both sectors are gapless along the second-order transition line between Haldane and dimerized phases while only the Ising sector is gapless along the transition line between NNN-Haldane and dimerized phases. The conformal embedding implies that each operator in the $\mathrm{SU}(2)_{2}$ field theory can be written as a product of operators in the Ising and free boson theories. It also implies that the finite size spectra are identical once certain selection rules are imposed. Here we give those selection rules, which were used to derive Table II of the Supplemental Material in Ref. [13]. We consider the case of open boundary conditions (OBC) on the spin chain with $N \gg 1$ sites, at the tri-critical point. The $\mathrm{SU}(2)_{2} \mathrm{WZW}$ model has three conformal towers, labeled by lowest energy states of $\operatorname{spin} j=0,1 / 2$, and 1 . The states in the spin- $j$ conformal tower have energies

$$
E_{j, n}=\frac{\pi v}{N}\left[-\frac{1}{16}+\frac{j(j+1)}{4}+n\right]
$$

for non-negative integer $n$. Excited states form multiplets of arbitrary spin, subject to the condition of being integer for $j=0,1$ and half-integer for $j=1 / 2$. In general, multiplets of a given spin and energy occur with integer degeneracies $\geqslant 1$. As argued in Ref. [13], the low-energy spectrum of the spin chain at the tricritical point is given by the $j=0$ conformal tower for $N$ even and the $j=1$ conformal tower for $N$ odd.
The Ising model has three conformal towers with energies

$$
E_{\mathrm{Ising}}=\frac{\pi v}{N}\left(-\frac{1}{48}+x^{\mathrm{Ising}}\right),
$$

where

$$
x^{\text {Ising }}=x+n
$$

$x=0,1 / 2$, or $1 / 16$, corresponding to the $I, \sigma$, and $\epsilon$ conformal towers and $n$ is a non-negative integer. The complete finite-size spectrum of the free boson model is

$$
E_{\text {boson }}=\frac{\pi v}{N}\left[-\frac{1}{24}+\frac{\left(S^{z}\right)^{2}}{2}+\sum_{n=1}^{\infty} m_{n} n\right],
$$

where $S^{z}$ labels the quantum number of the state and the $m_{n}$ are non-negative integers. This follows from the standard mode expansion for a periodic boson with $S^{z}$ the winding mode and the $m_{n}$ labeling excitations of the oscillator modes. Note that unlike the $\mathrm{SU}(2)_{2}$ and Ising cases, we have a simple explicit formula for all energies in the free boson case, labeled by $S^{z}$.

There are selection rules determining which Ising conformal towers can combine with boson states of various values of $S^{z}$. The three conformal towers of $\mathrm{SU}(2)_{2}$ correspond to the following selection rules:

$$
\begin{aligned}
& j=0:\left(S^{z}=\text { even integer, } I\right) \text { or }\left(S^{z}=\text { odd integer, } \epsilon\right), \\
& j=1:\left(S^{z}=\text { even integer, } \epsilon\right) \text { or }\left(S^{z}=\text { odd integer, } I\right), \\
& j=1 / 2:\left(S^{z}=\text { half-integer, } \sigma\right) .
\end{aligned}
$$

Using Eq. (9) and the known multiplicities of the Ising conformal towers we can work out the complete $\mathrm{SU}(2)_{2}$ conformal towers. Knowing the $S^{z}$ quantum numbers allows us to uniquely assign total spin quantum numbers $s$ to multiplets. (Of course, for this to be consistent, the number of states at a given level, $n$, for a given $S^{z}$ must be $\leqslant$ the number of states at the same level for smaller $S^{z}$. This turns out to always be the case.) Note that half-integer $S^{z}$ only occurring with $\sigma$ is consistent with the periodicity conditions discussed in Ref. [13]. These results lead to Tables I-V.

We can easily read off formulas for the energy of the lowest state of spin $s$ for any $s$ in each conformal tower. For the $j=0$ conformal tower, the lowest-energy state of $\operatorname{spin} s$ has

TABLE I. Details of $j=0$ conformal tower.

\begin{tabular}{rrrcc}
\hline \hline$n$ & $S^{z}$ & $m_{1}$ & $m_{2}$ & $x^{\text {Ising }}$ \\
\hline 0 & 0 & 0 & 0 & 0 \\
1 & \pm 1 & 0 & 0 & $1 / 2$ \\
1 & 0 & 1 & 0 & 0 \\
2 & \pm 2 & 0 & 0 & 0 \\
2 & \pm 1 & 1 & 0 & $1 / 2$ \\
2 & \pm 1 & 0 & 0 & $3 / 2$ \\
2 & 0 & 2 & 0 & 0 \\
2 & 0 & 0 & 1 & 0 \\
2 & 0 & 0 & 0 & 2 \\
\hline \hline
\end{tabular}


TABLE II. $j=0$ conformal tower, labeled by multiplicities of spin multiplets at each energy level.

\begin{tabular}{lccc}
\hline \hline$n$ & $s=0$ & $s=1$ & $s=2$ \\
\hline 0 & 1 & 0 & 0 \\
1 & 0 & 1 & 0 \\
2 & 1 & 1 & 1 \\
\hline \hline
\end{tabular}

energy

$$
\begin{aligned}
E & =\frac{\pi v}{N}\left(-\frac{1}{16}+\frac{s^{2}}{2}\right) \quad(s \text { even }) \\
& =\frac{\pi v}{N}\left(-\frac{1}{16}+\frac{s^{2}}{2}+\frac{1}{2}\right)(s \text { odd }) .
\end{aligned}
$$

For the $j=1$ conformal tower,

$$
\begin{aligned}
E & =\frac{\pi v}{N}\left(-\frac{1}{16}+\frac{s^{2}}{2}+\frac{1}{2}\right) \quad(s \text { even }), \\
& =\frac{\pi v}{N}\left(-\frac{1}{16}+\frac{s^{2}}{2}\right)(s \text { odd }) .
\end{aligned}
$$

These results are summarized in Table VI.

We have checked the validity of the conformal embedding by comparing the characters. The character for a conformal tower is the corresponding partition function:

$$
\chi_{x}=q^{-c / 12+x} \sum_{n=0}^{\infty} d_{n} q^{n} .
$$

Here, $q \equiv e^{-\pi v /(N T)}, c$ is the central charge $[3 / 2,1 / 2$, and 1 for $\mathrm{SU}(2)_{2}$, Ising, and free bosons, respectively] $x$ labels the conformal tower and the $d_{n}$ 's are the multiplicities.

For the boson conformal field theory, the characters are given by

$$
\chi(q)=q^{-\frac{1}{24}} \frac{1}{\varphi(q)} \sum_{S_{z}} q^{S_{z}^{2}},
$$

where $\varphi(q)=\prod_{n=1}^{\infty}\left(1-q^{n}\right)$ is the Euler function.

TABLE III. Details of $j=1$ conformal tower.

\begin{tabular}{rrrcc}
\hline \hline$n$ & $S^{z}$ & $m_{1}$ & $m_{2}$ & $x^{\text {Ising }}$ \\
\hline 0 & \pm 1 & 0 & 0 & 0 \\
0 & 0 & 0 & 0 & $1 / 2$ \\
1 & \pm 1 & 1 & 0 & 0 \\
1 & 0 & 1 & 0 & $1 / 2$ \\
1 & 0 & 0 & 0 & $3 / 2$ \\
2 & \pm 2 & 0 & 0 & $1 / 2$ \\
2 & \pm 1 & 2 & 0 & 0 \\
2 & \pm 1 & 0 & 1 & 0 \\
2 & \pm 1 & 0 & 0 & 2 \\
2 & 0 & 2 & 0 & $1 / 2$ \\
2 & 0 & 0 & 1 & $1 / 2$ \\
2 & 0 & 1 & 0 & $3 / 2$ \\
2 & 0 & 0 & 0 & $5 / 2$ \\
\hline \hline
\end{tabular}

TABLE IV. $j=1$ conformal tower, labeled by multiplicities of spin multiplets at each energy level.

\begin{tabular}{cccc}
\hline \hline$n$ & $s=0$ & $s=1$ & $s=2$ \\
\hline 0 & 0 & 1 & 0 \\
1 & 1 & 1 & 0 \\
2 & 1 & 2 & 1 \\
\hline \hline
\end{tabular}

If the sum is restricted to even values of $S_{z}$, this leads to

$$
\begin{aligned}
\chi_{\text {even }}^{\text {boson }}(q) & =q^{-\frac{1}{24}} \frac{1}{\varphi(q)} \sum_{n} q^{2 n^{2}} \\
& =q^{-\frac{1}{24}}\left(1+q+4 q^{2}+5 q^{3}+9 q^{4}+\cdots\right),
\end{aligned}
$$

while if the sum is restricted to odd values of $S_{z}$, this leads to

$$
\begin{aligned}
\chi_{\text {odd }}^{\text {boson }}(q) & =q^{-\frac{1}{24}} \frac{1}{\varphi(q)} \sum_{n} q^{(2 n-1)^{2} / 2} \\
& =q^{-\frac{1}{24}} q^{\frac{1}{2}} \frac{1}{\varphi(q)} \sum_{n} q^{2 n(n-1)} \\
& =q^{-\frac{1}{24}} q^{\frac{1}{2}}\left(2+2 q+4 q^{2}+6 q^{3}+12 q^{4}+\cdots\right) .
\end{aligned}
$$

Finally, if the sum is restricted to half-integer values of $S_{z}$, this leads to

$$
\begin{aligned}
\chi_{1 / 2}^{\text {boson }}(q) & =q^{-\frac{1}{24}} \frac{1}{\varphi(q)} \sum_{n} q^{\left(n-\frac{1}{2}\right)^{2} / 2} \\
& =q^{-\frac{1}{24}} q^{\frac{1}{8}} \frac{1}{\varphi(q)} \sum_{n} q^{\frac{n(n-1)}{2}} \\
& =q^{-\frac{1}{24}} q^{\frac{1}{8}}\left(2+2 q+4 q^{2}+6 q^{3}+12 q^{4}+\cdots\right) .
\end{aligned}
$$

For the Ising conformal field theory, the characters are given by (see Ref. [26], pp. 242-243):

$$
\begin{aligned}
\chi(q)= & q^{-\frac{1}{48}} q^{h_{r, s}} \frac{q^{-\frac{1}{48}} q^{-h_{r, s}}}{\varphi(q)} \\
& \times \sum_{n}\left[q^{\frac{(24 n+4 r-3 s)^{2}}{48}}-q^{\frac{(24 n+4 r+3 s)^{2}}{48}}\right],
\end{aligned}
$$

where $h_{r, s}=\frac{(4 r-3 s)^{2}-1}{48}$ and where $(r, s)=(1,1)$ for $I,(r, s)=$ $(2,1)$ for $\epsilon$, and $(r, s)=(1,2)$ for $\sigma$.

This leads to the following characters for $I, \epsilon$, and $\sigma$ :

$$
\begin{aligned}
\chi_{I}^{\text {Ising }}(q) & =q^{-\frac{1}{48}} \frac{q^{-\frac{1}{48}}}{\varphi(q)} \sum_{n}\left[q^{\frac{(24 n+1)^{2}}{48}}-q^{\frac{(24 n+7)^{2}}{48}}\right] \\
& =q^{-\frac{1}{48}}\left(1+q^{2}+q^{3}+2 q^{4}+2 q^{5}+\cdots\right),
\end{aligned}
$$

TABLE V. $j=1 / 2$ conformal tower, labeled by multiplicities of spin multiplets at each energy level.

\begin{tabular}{lcc}
\hline \hline$n$ & $s=1 / 2$ & $s=3 / 2$ \\
\hline 0 & 1 & 0 \\
1 & 1 & 1 \\
2 & 2 & 2 \\
\hline \hline
\end{tabular}


TABLE VI. Lowest excitation energy with spin $s$ for both $j=0$ and 1 conformal towers.

\begin{tabular}{lllllll}
\hline \hline $\mathrm{s}$ & 0 & 1 & 2 & 3 & 4 & 5 \\
\hline$\left(E-E_{0}\right) N / \pi v, j=0$ & 0 & 1 & 2 & 5 & 8 & 13 \\
$\left(E-E_{0}\right) N / \pi v, j=1$ & 1 & 0 & 2 & 4 & 8 & 12 \\
\hline \hline
\end{tabular}

$$
\begin{aligned}
\chi_{\epsilon}^{\text {Ising }}(q) & =q^{-\frac{1}{48}} q^{\frac{1}{2}} \frac{q^{-\frac{1}{48}} q^{-\frac{1}{2}}}{\varphi(q)} \sum_{n}\left[q^{\frac{(24 n+5)^{2}}{48}}-q^{\frac{(24 n+1)^{2}}{48}}\right] \\
& =q^{-\frac{1}{48}} q^{\frac{1}{2}}\left(1+q+q^{2}+q^{3}+2 q^{4}+2 q^{5}+\cdots\right), \\
\chi_{\sigma}^{\text {Ising }}(q) & =q^{-\frac{1}{48}} q^{\frac{1}{16}} \frac{q^{-\frac{1}{48}} q^{-\frac{1}{16}}}{\varphi(q)} \sum_{n}\left[q^{\frac{(24 n-2)^{2}}{48}}-q^{\frac{(24 n+10)^{2}}{48}}\right] \\
& =q^{-\frac{1}{48}} q^{\frac{1}{16}}\left(1+q+q^{2}+2 q^{3}+2 q^{4}+3 q^{5}+\cdots\right) .
\end{aligned}
$$

For the SU(2) 2 conformal field theory, the characters for a given $j$ are given by (see Ref. [26], p. 585)

$$
\chi(q)=q^{-\frac{1}{16}} q^{\frac{j(j+1)}{4}} \frac{\sum_{n}(2 j+1+8 n) q^{n(2 j+1+4 n)}}{\sum_{n}(1+4 n) q^{n(1+2 n)}} .
$$

This leads to the following characters for $j=0, \frac{1}{2}$ and 1 :

$$
\begin{aligned}
& \chi_{j=0}^{\mathrm{SU}(2)_{2}}(q)=q^{-\frac{1}{16}}\left(1+3 q+9 q^{2}+15 q^{3}+30 q^{4}+\cdots\right), \\
& \chi_{j=\frac{1}{2}}^{\mathrm{SU}(2)_{2}}(q)=q^{\frac{1}{8}}\left(2+6 q+12 q^{2}+26 q^{3}+48 q^{4}+\cdots\right), \\
& \chi_{j=1}^{\mathrm{SU}(2)_{2}}(q)=q^{\frac{7}{16}}\left(3+4 q+12 q^{2}+21 q^{3}+43 q^{4}+\cdots\right) .
\end{aligned}
$$

By expanding all these characters to order 100 or higher, we have checked that the following relations hold:

$$
\begin{aligned}
\chi_{\text {even }}^{\text {boson }}(q) \chi_{I}^{\text {Ising }}(q)+\chi_{\text {odd }}^{\text {boson }}(q) \chi_{\epsilon}^{\text {Ising }}(q) & =\chi_{j=0}^{\mathrm{SU}(2)_{2}}(q), \\
\chi_{\text {even }}^{\text {boson }}(q) \chi_{\epsilon}^{\text {Ising }}(q)+ & \chi_{\text {odd }}^{\text {boson }}(q) \chi_{I}^{\text {Ising }}(q)=\chi_{j=1}^{\mathrm{SU}(2)_{2}}(q), \\
\chi_{1 / 2}^{\text {boson }}(q) \chi_{\sigma}^{\text {Ising }}(q) & =\chi_{j=\frac{1}{2}}^{\mathrm{SU}(2)_{2}}(q)
\end{aligned}
$$

corresponding to Eq. (10).

\section{B. DMRG}

Most of the numerical results in this paper have been obtained with density matrix renormalization group (DMRG) algorithm. The only exception is the calculation of the Berry phase that has been done on small rings by exact diagonalization. In this section, we provide some technical details on the DMRG algorithm we have used.

First of all, we have used the matrix product state formulation of DMRG, and therefore the proper name would be variational MPS. The code consists of four parts:

(1) Infinite-size DMRG. The system size grows from 2 to $N$ by inserting two-site matrix product operator (MPO) in the middle of the chain and by diagonalizing the corresponding effective Hamiltonian. Everything on the left and on the right of the MPO is effectively described by the left and right environments. The singular value decomposition of the eigenvector produces left and right normalized on-site tensors. These tensors are multiplied with the corresponding environments in order to update them and at the same time to increase the size of each environment by one. For an odd total number of spins $N$, the same procedure is performed until the system reaches a size of $N-1$, in which case only one tensor is multiplied to the environment. Assuming without loss of generality that the left environment was updated and therefore contains an effective basis for $(N-1) / 2$ spins, one can reuse the right environment for $(N-3) / 2$ spins and insert the local Hamiltonian for two additional spins to reach a system size with $\mathrm{N}$ odd. In this part of the code, we usually keep 44 singular values. However, close to the critical lines, we increase this number to 66 for systems larger than $N \geqslant 300$ spins. The infinite-size DMRG provides a good starting point for the remaining parts of the code.

(2) The warm-up function consists of an incomplete sweep. Sweeping from the middle of the chain to its right end we update local tensors site by site and increase the number of kept states by a factor 1.5. Sweeping back from the right end to the left one we again increase the number of states by the same factor. Therefore in the end of the warm-up the number of kept states is 100 (or 150 for 66 states in infinite-size DMRG).

(3) The "main body" of the algorithm is sweeping from left to right and back locally updating the tensors. We usually perform 6 or 7 sweeps for open boundary conditions and up to 16 sweeps for periodic chains. We keep up to 700 singular values for $N<200$ and up to 900 states for larger systems. During the first 6 or 7 sweeps we increase the number of states linearly up to its maximal value. For periodic chains we continue to jiggle the wave-function by decreasing and increasing the number of states until the convergence is reached. The traditional formulation of the variational MPS imply "one-site" DMRG, where the effective Hamiltonian diagonalized at each iteration represents a single spins in its left and right environments. Since the dimerized phase has two spins per unit cell, we implement a two-site routine, which turns out to be significantly more stable and to converge faster, despite the obvious growth of complexity by a factor $(2 S+1)^{2}=9$. Roughly speaking, the number of kept states 700 and 900 for two-site DMRG is equivalent to 1210 and 1560 for the one-site routine, although there is no simple one-to-one correspondence.

(4) During the "final sweep," we do not increase the number of states anymore, but at each iteration we measure the set of local observables such as on-site magnetization, nearestneighbor spin-spin correlations, and entanglement entropy. The left and right normalized tensors and vectors of the Schmidt decomposition are stored and used later in order to calculate the observables which involve more than two spins (energy in the middle of the chain, long-range correlations, structure factor, etc.) or to extract the entanglement spectrum.

A significant role in the successful convergence is played by an efficient representation of the Hamiltonian (1) in terms MPO. The MPO is a four-dimensional tensor with two physical and two auxiliary legs. The complexity of the algorithm is proportional to the dimension of the latest. The straightforward MPO representation of the $J_{1}-J_{2}-J_{3}$ model has a bond dimension $d=17\left(3+3\right.$ for $J_{1}$ and $J_{2}$ terms, 9 for $J_{3}$ interaction, 1 for unity matrix, and 1 for magnetic field or so-called "full term"). Using the spin commutation relations, this number can be reduced to $d=14$. Below we show a different approach that allows to reduce the bond dimension to $d=8$. 
The efficient MPO representation naturally appears when the $J_{3}$ term is rewritten in terms of quadrupolar operators:

$$
\sum_{i} \sum_{\alpha, \beta=x, y, z} J_{3} S_{i-1}^{\alpha} Q_{i}^{\alpha \beta} S_{i+1}^{\beta}
$$

where

$$
Q_{i}^{\alpha \beta}=S_{i}^{\alpha} S_{i}^{\beta}+S_{i}^{\beta} S_{i}^{\alpha}
$$

Generally speaking, $Q$ is not a traceless tensor, and therefore it is not a quadrupolar operator, but let us keep the $Q$ notation for simplicity. Combining the new expression for the $J_{3}$ term with the $J_{2}$ term, one obtains the Hamiltonian in the following form:

$$
\begin{aligned}
H= & \sum_{i} J_{1} \mathbf{S}_{i} \cdot \mathbf{S}_{i+1} \\
& +\sum_{i} \sum_{\alpha, \beta=x, y, z} S_{i-1}^{\alpha}\left(J_{2} \delta^{\alpha \beta}+J_{3} Q_{i}^{\alpha \beta}\right) S_{i+1}^{\beta} .
\end{aligned}
$$

The sum in brackets can be written in matrix form as

$$
\left(\begin{array}{ccc}
J_{2} I+J_{3} Q^{x x} & J_{3} Q^{x y} & J_{3} Q^{x z} \\
J_{3} Q^{x y} & J_{2} I+J_{3} Q^{y y} & J_{3} Q^{y z} \\
J_{3} Q^{x z} & J_{3} Q^{y z} & J_{2} I+J_{3} Q^{z z}
\end{array}\right),
$$

where $I$ is a $d \times d$ unity matrix, with $d=2 S+1$. In terms of rescaled lowering and raising operators $S_{i}^{ \pm}=\left(S_{i}^{x} \pm i S_{i}^{y}\right) / \sqrt{2}$, the MPO tensor reads

$$
H_{i}=\left(\begin{array}{ccccccccc}
I & & & & & & \\
S_{i}^{-} & & & & & & \\
S_{i}^{+} & & & & & & \\
S_{i}^{z} & & & & & & \\
& J_{2} I+J_{3} Q^{+-} & J_{3} Q^{--} & J_{3} Q^{-z} & & & \\
& J_{3} Q^{++} & J_{2} I+J_{3} Q^{+-} & J_{3} Q^{+z} & & & \\
& J_{3} Q^{+z} & J_{3} Q^{-z} & J_{2} I+J_{3} Q^{z z} & & & \\
& J_{1} S_{i}^{+} & J_{1} S_{i}^{-} & J_{1} S_{i}^{z} & S_{i}^{+} & S_{i}^{-} & S_{i}^{z} & I
\end{array}\right)
$$

above a few low-lying singlet excitations for systems with $N>$ 30, as shown in Ref. [13]. The picture is more complicated when the $J_{1}$ coupling is absent. The bonds close to each edge can be excited to a triplet state with a very low energy. The singlet bulk excitations are below the edge excitations for very large system sizes $N>300$, see Fig. 20. When bulk and edge excitations are close enough, they can be distinguished by looking at the excitation energy as a function of iterations. The energies obtained by diagonalizing the effective Hamiltonian with an MPO located in the middle of the chain are related to the bulk excitations, while minima in energies around the end of each half-sweep correspond to edge excitations. In Fig. 3, we provide an example for the $J_{2}-J_{3}$ chain with $N=300$ spins.

\section{PHASE DIAGRAM}

\section{A. Dimerization}

The natural order parameter to identify the dimerized phase is the dimerization parameter defined by $D=\mid\left\langle\mathbf{S}_{i} \cdot \mathbf{S}_{i+1}-\mathbf{S}_{i}\right.$. $\left.\mathbf{S}_{i-1}\right\rangle \mid$, where $(i, i+1)$ is the central bond of an open chain. Figure 4 shows numerical results for the dimerization of a chain with $N=150$ sites as a function of $J_{3}$ obtained by variational MPS.

In large systems $(N=120,150)$ and close to the first-order phase transition, the variational MPS algorithm suffers from a kind of hysteresis: the algorithm converges to the first excited state instead of the ground state. This results in an unphysical jump in the energy curve and in an abrupt change of dimerization before the actual phase transition. These results were discarded when discussing the nature of the phase transition, and Fig. 4 presents only dimerization curves for which the finite-size energy is continuous. 

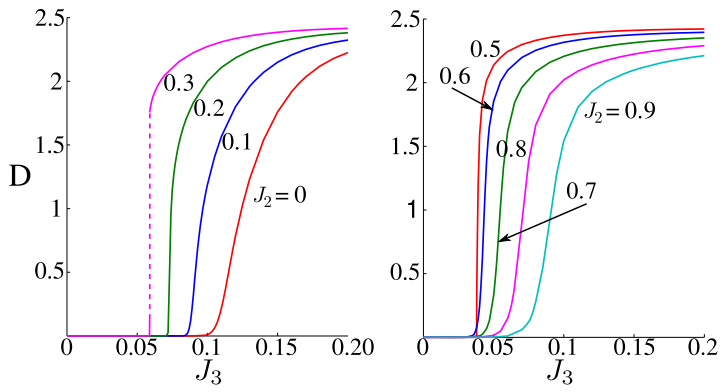

FIG. 4. Finite-size $(N=150)$ dimerization as a function of $J_{3}$ for $0 \leqslant J_{2} \leqslant 0.3$ (left) and $0.5 \leqslant J_{2} \leqslant 0.9$ (right). The dashed line indicates a first-order phase transition.

In order to determine the boundary of the dimerized phase in the thermodynamic limit, we have performed a finite-size extrapolation for chains with $N=30,60,90,120$, and 150 sites. A chain is in the dimerized phase if the dimerization stays finite for $N \rightarrow \infty$, which we associate with a convex curve in a $\log -\log$ plot. By contrast, a concave scaling curve leads to a vanishing dimerization in the thermodynamic limit and therefore means that the system is in the Haldane or NNN-Haldane phase. The phase transition then corresponds to a straight line in the scaling. Some examples of finite-size scaling are shown in Fig. 5. A smooth change of the scaling curvature implies that the dimerization curve is continuous in the thermodynamic limit Figs. 5(a) and 5(c), while a first-order phase transition with a finite jump in the dimerization curve leads to an abrupt change from concave to convex scaling at the critical point Fig. 5(b). The investigation of dimerization has led to a precise determination of the transition line, and of the nature of the phase transition (continuous or first order) except in the vicinity of the end point of the continuous transition between the Haldane phase and the dimerized phase (see section E).

\section{B. Ground-state energy}

In view of the hysteretic behavior of the system, and to complete the phase diagram in regions where the groundstate energy appears to be discontinuous, we have carefully investigated the behavior of the energy in the vicinity of the transition lines. An estimate of the ground-state energy in the
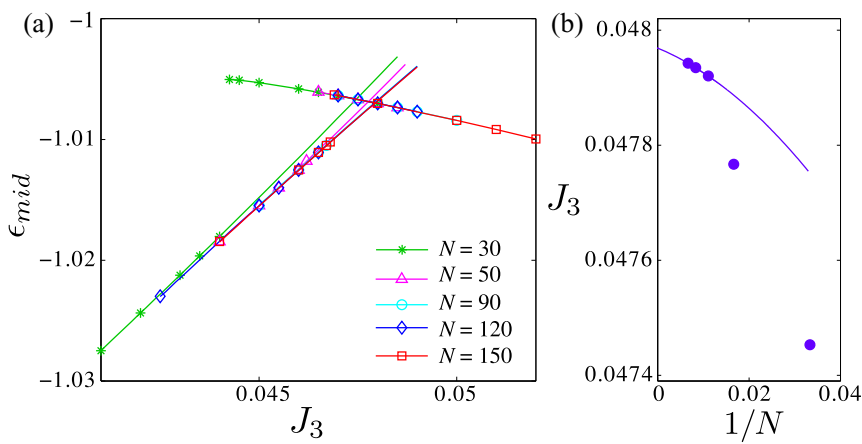

FIG. 6. (a) Energy of the central bonds for $J_{2}=0.4$ as a function of $J_{3}$ for finite-size chains with $N=30,60,90,120$, and 150 sites. Solid lines are polynomial fits. The kink was created by letting the two fits cross. b) Position of the kink in $\epsilon_{\text {mid }}$ as a function of size. The fitting curve is a quadratic function in $1 / N$.

thermodynamic limit is provided by the value of the energy of the central bonds:

$$
\epsilon_{\text {mid }}=\epsilon_{1}+\epsilon_{2}+\epsilon_{3}
$$

where

$$
\begin{aligned}
& \epsilon_{1}=\frac{J_{1}}{2}\left\langle\mathbf{S}_{i-1} \cdot \mathbf{S}_{i}+\mathbf{S}_{i} \cdot \mathbf{S}_{i+1}\right\rangle, \\
& \epsilon_{2}=J_{2}\left\langle\mathbf{S}_{i-1} \cdot \mathbf{S}_{i+1}\right\rangle, \\
& \epsilon_{3}=J_{3}\left\langle\left(\mathbf{S}_{i-1} \cdot \mathbf{S}_{i}\right)\left(\mathbf{S}_{i} \cdot \mathbf{S}_{i+1}\right)+\text { H.c. }\right\rangle,
\end{aligned}
$$

and where $(i, i+1)$ is the central bond. The dependence on $J_{3}$ of $\epsilon_{\text {mid }}$ for chains with $N=30,60,90,120$, and 150 sites for $J_{2}=0.4$ is presented in Fig. 6(a). The energy curves are discontinuous due to the edge effects and due to hysteresis of the variational MPS algorithm for $N=120$ and 150. In order to determine as precisely as possible the location of the first-order phase transition in the thermodynamic limit, we have extrapolated the lines until they cross. Then, a finite-size scaling of the position of the kink is presented in Fig. 6(b).

For $0.25 \leqslant J_{2} \leqslant 0.45$, the ground-state energy and the dimerization parameter lead to the same estimate for the location of the phase transition. For larger next-nearestneighbor coupling, the kink disappears for small clusters but it is still present in large chains (see Fig. 7). The phase transition line continues towards small $J_{3}$ and end up at $J_{3}=0$ and
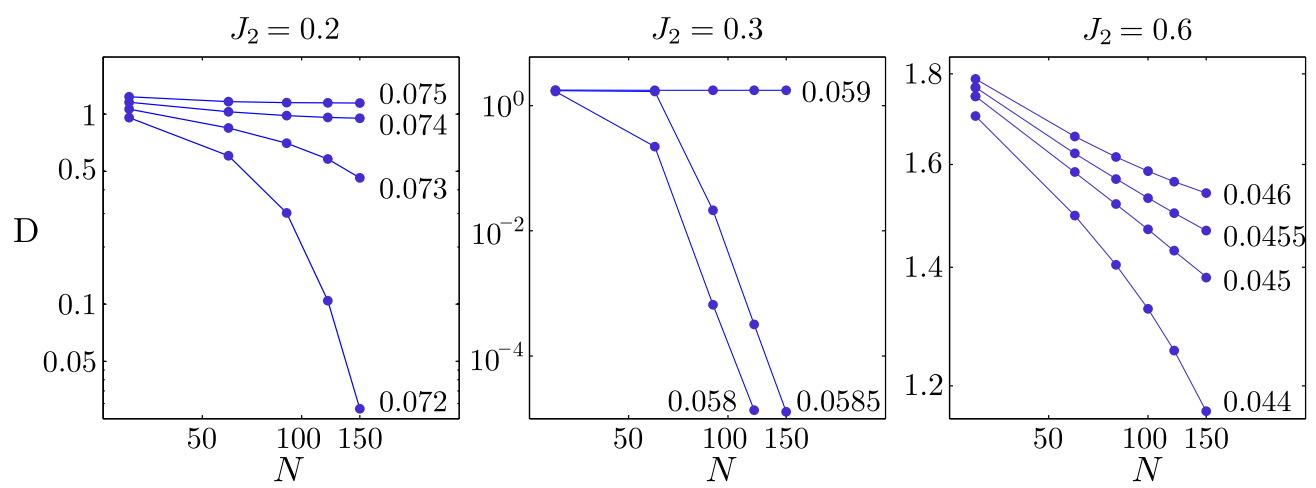

FIG. 5. Finite-size scaling of the dimerization parameter for $J_{2}=0.2,0.3$, and 0.6 . The value of $J_{3}$ is attached to each curve. The phase transition is continuous at $J_{2}=0.2$ and 0.6. The abrupt change of scaling at $J_{2}=0.3$ indicates a first-order phase transition. 

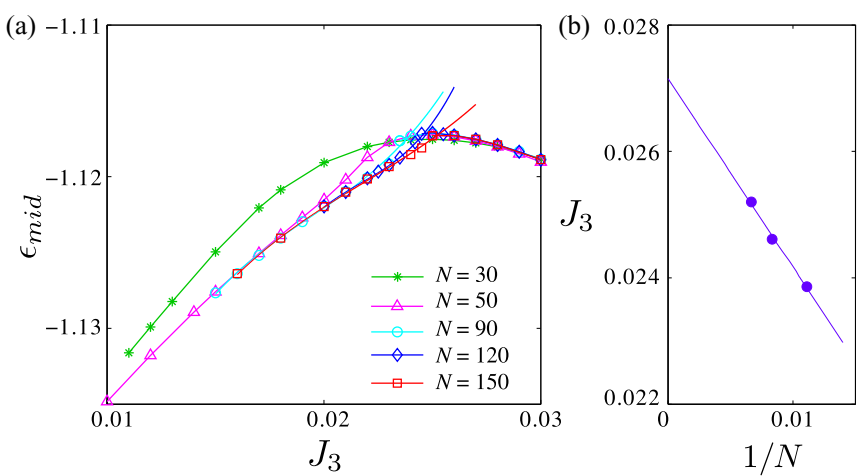

FIG. 7. Same as Fig. 6 for $J_{2}=0.6$.

$J_{2}=0.75$, close to the value $0.0744(4)$ obtained by Kolezhuk et al. $[8,18]$.

In order to confirm the location of the continuous phase transition deduced from the dimerization parameter, we have calculated the second derivative of $\epsilon_{\text {mid }}$ with respect to $J_{3}$. Examples for $N=90$ and 150 are shown in Fig. 8. A kink in the energy implies a divergence of its second derivative. Besides divergences, one can see the appearance of pronounced minima, which agree with the continuous finitesize phase transitions found with the dimerization parameter. The finite-size effect slightly increase with increasing $J_{2}$. The positions of the minima are in good agreement with the phase boundaries found with the dimerization parameter for $J_{2} \leqslant 0.2$ and $J_{2} \geqslant 0.5$ (see scaling comparison on Fig. 14).

The kink across the first-order phase transition between the Haldane phase and the NNN-Haldane phase is only visible for nonzero value of $J_{3}$. At $J_{3}=0$, the first derivative of the ground-state energy with respect to $J_{2}$ is not discontinuous for finite chains, as reported before [8]. Although the second derivative is not divergent, the position of its minimum at $J_{2}=0.75$ coincides with the extrapolation of the critical line deduced from the kink in the energy for $J_{3}>0$.

\section{Entanglement spectrum}

As mentioned in the introduction, the Haldane phase of the spin-1 chain is an example of a symmetry protected topological phase in one dimension [27]. It is distinct from the topologically trivial NNN-Haldane and dimerized phases, and it can be characterized by the finite value of the string order parameter, a criterion already used for the $J_{1}-J_{2}$ model [8]. More recently, it has been proposed to characterize topological phases by their entanglement spectrum, obtained by dividing the system into two parts, tracing out one of them, and diagonalizing the reduced density matrix of the remaining part [28-30]. This creates artificial edges without breaking the inversion symmetry.

In the present case, a system with open ends may be partitioned across a certain bond and the wave function can be then Schmidt decomposed as

$$
|\Psi\rangle=\sum_{\alpha} \lambda_{\alpha}\left|L_{\alpha}\right\rangle\left|R_{\alpha}\right\rangle,
$$

where $\left|L_{\alpha}\right\rangle$ and $\left|R_{\alpha}\right\rangle$ are orthonormal basis vectors of the left and right parts. In variational MPS, the Schmidt values $\lambda_{\alpha}$ are obtained naturally at each iteration. Now, the multiplicity of the Schmidt values is related to the number of edge states that appear due to partitioning: Any topologically nontrivial phase is characterized by at least twofold degeneracy. Pollmann et al. [17] have shown that the Haldane phase of $S=1$ chains is characterized by a twofold degeneracy of the entanglement spectrum.
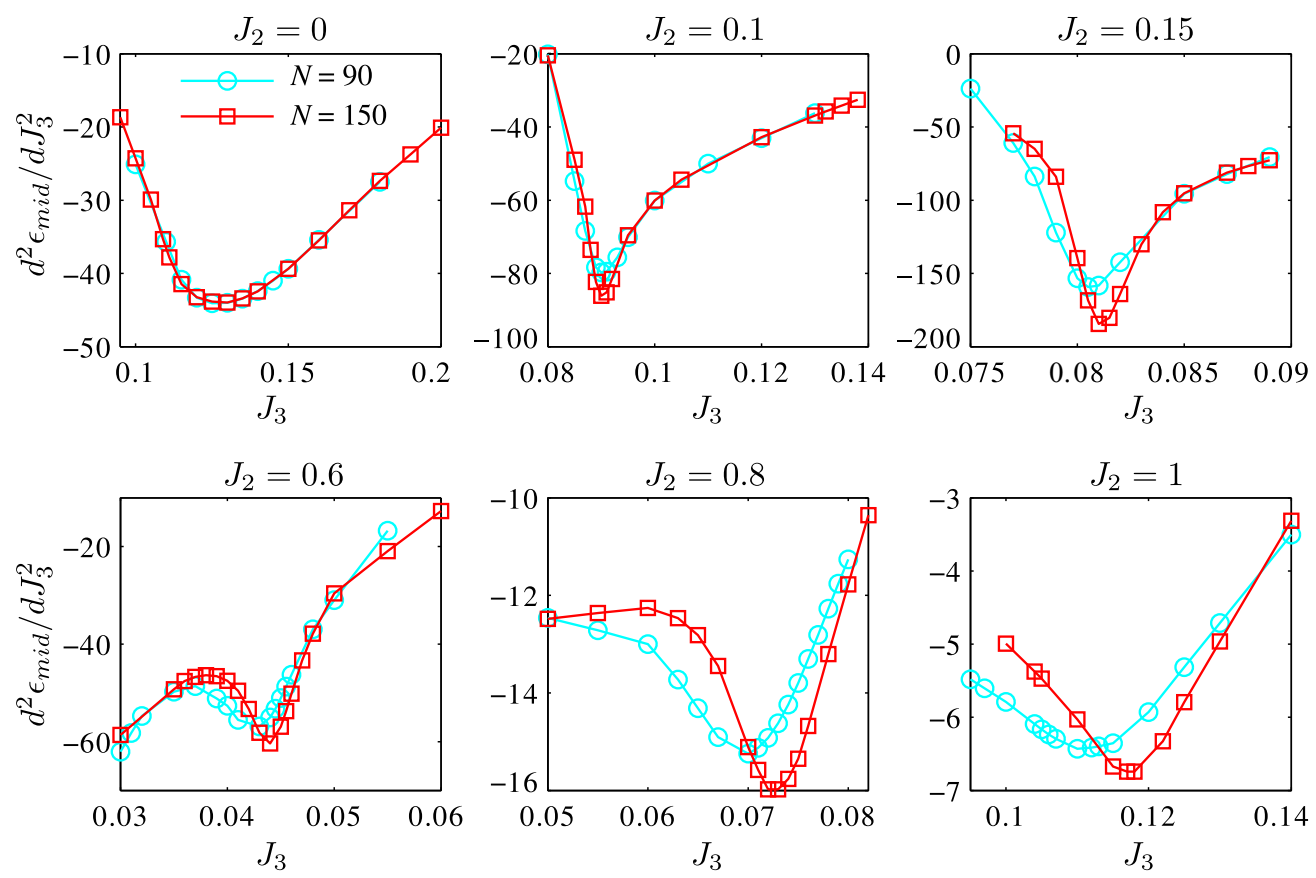

FIG. 8. (Top) Second derivative of $\epsilon_{\text {mid }}$ with respect to $J_{3}$ for $N=90,150$ and $J_{2}=0,0.1,0.15$ across continuous phase transition between Haldane and dimerized phases. (Bottom) Second derivative of $\epsilon_{\text {mid }}$ for $N=90,150$ and $J_{2}=0.6,0.8,1$ across the transition line between NNN-Haldane and dimerized phases. 


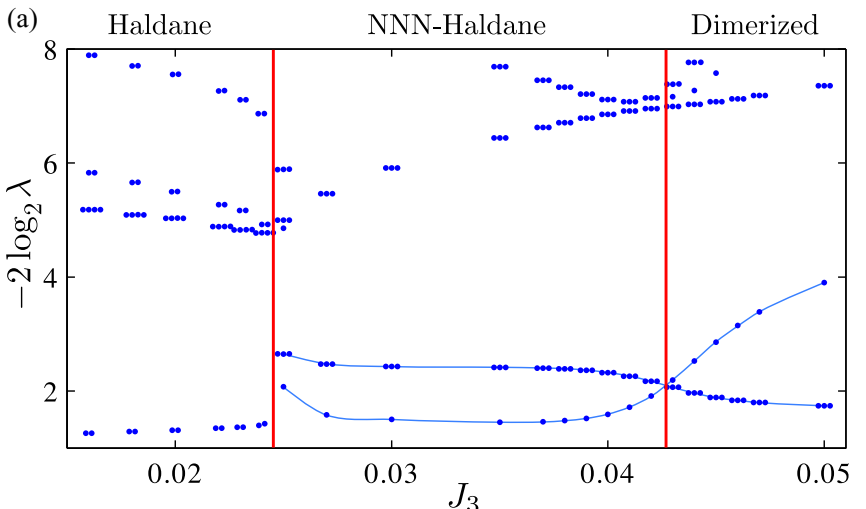

(b)

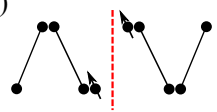

(c)

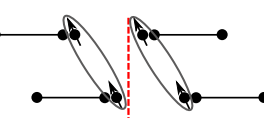

(d)

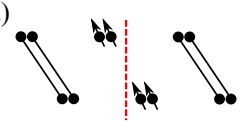

FIG. 9. (a) Entanglement spectrum for an open chain with $N=$ 150 sites as a function of $J_{3}$ (only the lower part of the spectrum is shown). The dots show the multiplicity of the Schmidt values. The plot for $J_{2}=0.6$ is shown here as an example. (b)-(d) VBS sketches of the artificial edges created by the bipartition of the chain in Haldane, NNN-Haldane, and dimerized phase, respectively. (b) The black arrows at each edge stand for two free spins $1 / 2$, which form a singlet, leading to a twofold degenerate entanglement spectrum. (c) The two spin-1/2 created at each edge couple with each other, which is represented as a gray ellipse. There are no edge states, and the entanglement spectrum is nondegenerate. (d) The edge spins are spins 1 and form a singlet, which leads to a threefold degenerate entanglement spectrum.

An example of finite-size entanglement spectrum containing all three phases is shown in Fig. 9. Three VBS (valence bond solid) sketches are attached in order to show how edge states are formed in each phase. In complete agreement with previous works, the entanglement spectrum in the Haldane phase is twofold degenerate, the edge states being spins $1 / 2$. By contrast, the entanglement spectrum is nondegenerate in the NNN-Haldane phase because there are no edge states. For the dimerized phase, it depends where the system is cut. For a system with open boundary conditions and an even number of sites, the ground state is non degenerate and consists of alternating strong and weak bonds. If the system is cut in the middle of a weak bond, no edge states appear, and the entanglement spectrum is non degenerate. However, if the system is cut on a strong bond, i.e., on a bond which is essentially a singlet made of two spins 1, as done in Fig. 9, the entanglement spectrum is threefold degenerate because spin-1 edge states are created, and the NNN-Haldane phase can be distinguished from the dimerized phase. In small systems an intermediate phase with a three fold degenerate entanglement spectrum and a low-lying nondegenerate level appears between Haldane and NNN-Haldane phases. This phase disappears for larger system sizes and is a thus a finite-size effect.

The resulting phase diagram is shown in Fig. 10. It is consistent with other approaches, but finite-size effects are strong, especially for the transition between the NNN-Haldane phase and the dimerized phase. (a)

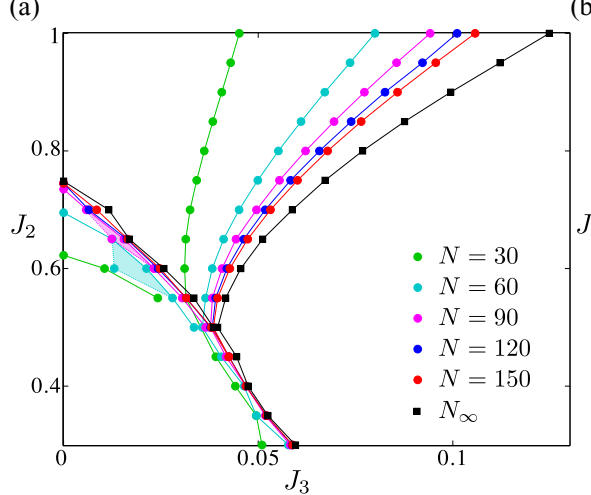

(b)

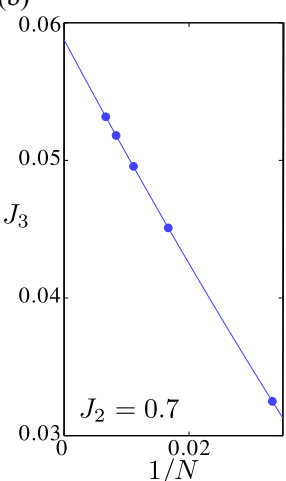

FIG. 10. (a) Phase boundaries deduced from the entanglement spectrum for chains with $N=30,60,90,120$, and 150 sites, and after finite-size scaling $\left(N_{\infty}\right)$. Shaded area: intermediate phase for $N=60$ and 90. (b) Example of finite-size scaling for $J_{2}=0.7$ with a quadratic fit.

\section{Berry phase}

Another powerful tool to characterize topologically nontrivial phases is the Berry phase [24] that can be defined for any Hamiltonian $H(\phi)$, which depends periodically on a parameter $\phi$. If $|G S(\phi)\rangle$ is a single-valued ground state of $H(\phi)$, the Berry connection is given by $A(\phi)=\left\langle G S(\phi)\left|\partial_{\phi}\right| G S(\phi)\right\rangle$, and the Berry phase is the integration of the Berry connection over a loop:

$$
i \gamma=\oint A(\phi) d \phi
$$

It was proposed by Hatsugai et al. [25] to use the angle $\phi$ of the twist of the transverse component of the spin-spin interaction on a given bond $(i, j)$

$$
S_{i}^{+} S_{j}^{-}+S_{i}^{-} S_{j}^{+} \rightarrow e^{i \phi} S_{i}^{+} S_{j}^{-}+e^{-i \phi} S_{i}^{-} S_{j}^{+} .
$$

Then the number of valence bond singlets $B_{i j}$ on the bond $(i, j)$ is related to the Berry phase by

$$
\gamma=B_{i j} \pi, \quad \bmod (2 \pi) .
$$

In other words, the Berry phase gives access to the parity of the number of valence-bond singlets on a given bond.

Previous studies of the Berry phase in spin systems have demonstrated that topological phase transitions can be reliably captured when the applied twist at $\phi=\pi$ is equivalent to antiperiodic boundary conditions for the transverse component of the interaction. To fulfill this requirement, three bonds must be simultaneously twisted as shown in Fig. 11(a). The twist applied on a bond $(i, i+1)$ implies that the transverse component of the interaction in the initial Hamiltonian is changed in all terms where the term $\mathbf{S}_{i} \cdot \mathbf{S}_{i+1}$ appears, i.e. both in the $J_{1}$ and $J_{3}$ terms. The twist of an $(i, i+2)$ bond changes only the $J_{2}$ term.

In the dimerized phase, there is no singlet on next-nearest neighbor bonds $(i-1, i+1)$ and $(i, i+2)$, while bonds $(i, i+$ 1) have either zero or two singlets. So the Berry phase $\gamma$, which is defined only up to $2 \pi$, is equal to zero. In the NNN-Haldane phase, the bonds $(i-1, i+1)$ and $(i, i+2)$ contain one spin$1 / 2$ singlet each and there is no singlet on the link $(i, i+1)$, 
(a)

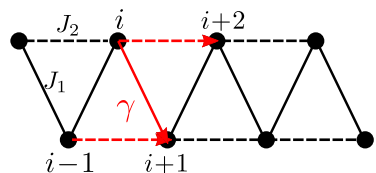

(b)

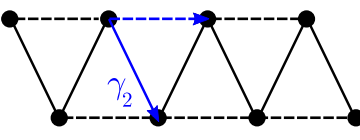

FIG. 11. (a) Berry phase applied on three bonds to be consistent with antiperiodic boundary conditions for the transverse component of the spin-spin interaction at $\phi=\pi$. This Berry phase $\gamma=\pi$ in the Haldane phase and $\gamma=0$ otherwise. (b) Berry phase applied on two bonds to distinguish the dimerized phase $\left(\gamma_{2}=0\right)$ from the Haldane and NNN-Haldane phases $\left(\gamma_{2}=\pi\right)$.

so that $\gamma=0$ as in the previous case. By contrast, the Berry phase is equal to $\pi$ in the Haldane phase, in which there is one VBS singlet on the bond $(i, i+1)$ and no VBS singlet on the bonds $(i-1, i+1)$ and $(i, i+2)$.

We have calculated the Berry phase $\gamma$ for chains with periodic boundary conditions using exact diagonalizations. The results for finite sizes are presented in Figs. 12(a)-12(c), and the finite-size scaling based on chains of length $N=$ $8,10,12$, and 14 sites in the interval $0 \leqslant J_{3} \leqslant 0.25$ is shown in Fig. 12(d). The results from the finite-size extrapolation are also included in Fig. 12(a). Systems close to the firstorder phase transition have strong finite-size effects, and no meaningful extrapolation could be performed with only four points. There is also a clear indication of an even-odd effect: the scaling for $N=8,12, \ldots, 4 k$ is different from the one for $N=$ $10,14, \ldots, 2(2 k+1)$. For $J_{2} \geqslant 0.3$, the results for $N=12$ (the largest accessible chain with an even number of spin pairs) is taken as the Berry phase estimate of the phase boundary. Quite remarkably, the finite-size results for such small chains are already very close to the phase boundaries obtained in the thermodynamic limit with other techniques (see Fig. 14 and 15).

When the twist used to define the Berry phase does not correspond to anti-periodic boundary conditions at $\phi=\pi$, the Berry phase can still reflect some local properties of the system and capture phase transitions. To distinguish the dimerized phase from the NNN-Haldane phase, we propose to define the Berry phase by twisting two links as shown in Fig. 11(b). Similarly to what was done for the three-bond Berry phase, we apply the twist on two bonds $(i, i+1)$ and $(i, i+2)$ simultaneously. In the Haldane phase, there is only one VBS singlet on the bond $(i, i+1)$, in the NNN-Haldane phase one VBS singlet on the $(i, i+2)$ bond, and in both cases $\gamma_{2}=\pi$. In the dimerized phase the bond $(i, i+1)$ contains either zero or two singlets while the $(i, i+2)$ bonds have no singlets, and the Berry phase $\gamma_{2}=0$. The finite-size results for $N=12$ and 14 are shown in Fig. 13. Qualitatively, this Berry phase gives the same phase boundaries as the dimerization parameter, which is also shown as a reference line. There is a strong finite-size effect however, and the extrapolation to the thermodynamic limit requires bigger system sizes.

\section{E. Comparison}

To show that all approaches presented above capture essentially the same phase diagram, we provide examples of comparative finite-size scaling (Fig. 14) and phase diagrams obtained with different criteria (Fig. 15). We compare the phase (a)

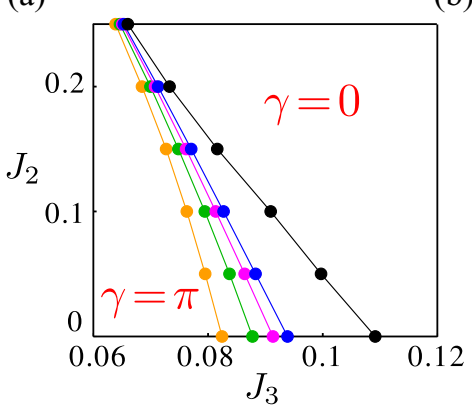

(d)

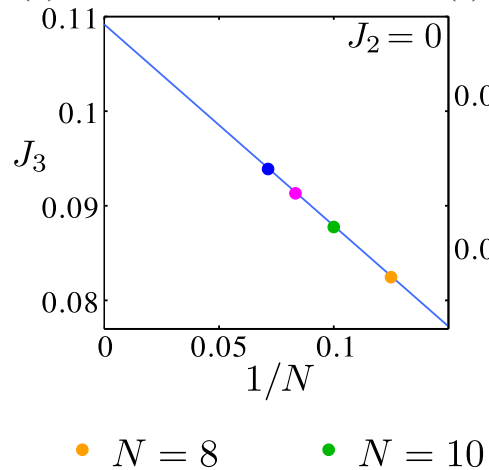

(e) (b)

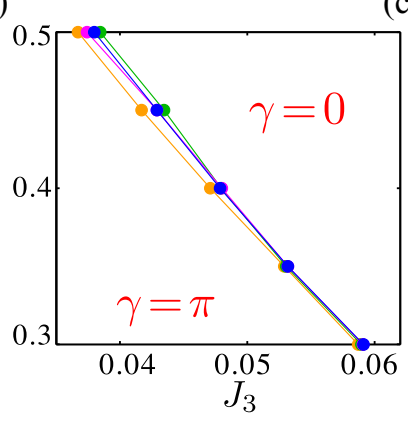

(c)

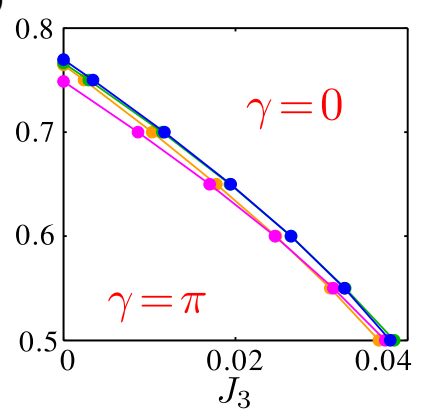

(f)

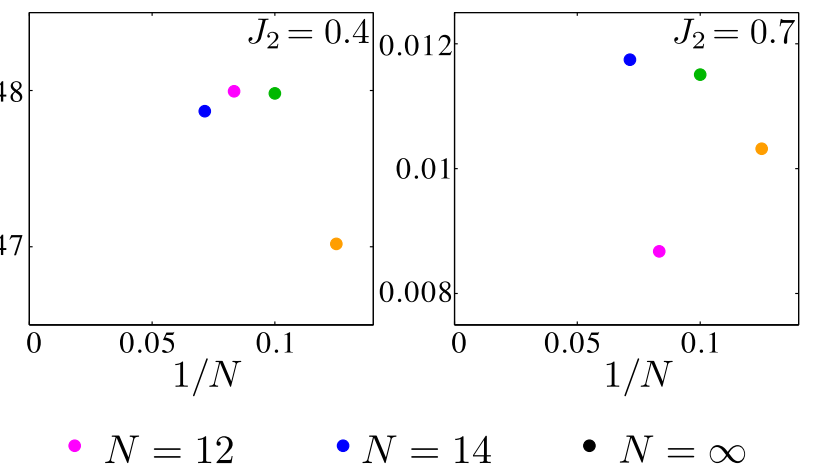

FIG. 12. Results of the Berry phase calculation in a periodic chain with $N=8,10,12,14$. (Top) Finite size phase transitions captured by the Berry phase for $J_{2}$ in the range (a) $0 \leqslant J_{2} \leqslant 0.25$, (b) $0.3 \leqslant J_{2} \leqslant 0.5$, and (c) $0.5 \leqslant J_{2} \leqslant 0.78$. The results of finite-size extrapolation are shown in (a) as a black line. (Bottom) Size dependence of the boundaries deduced from Berry phase. (d) Finite-size scaling for $J_{2}=0$ performed with a cubic fit in $1 / N$. (e) and (f) Examples of strong finite-size effects that do not allow one to make a finite-size extrapolation. 


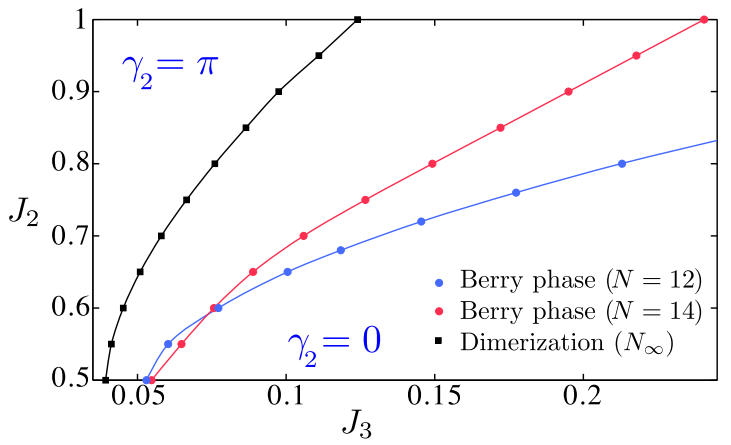

FIG. 13. Phase transition obtained by the $\gamma_{2}$ Berry phase for periodic chains with $N=12$ (blue circles) and $N=14$ (red circles) sites. The phase transition, obtained with finite-size scaling of the dimerization parameter (black squares) is shown as a reference.

boundaries deduced from the dimerization, energy, entanglement spectra, and Berry phase (Fig. 15). For $J_{2}=0$, the second derivative in the energy gives a phase boundary different from the one obtained with the dimerization parameter. Except for this point, the two boundaries are in rather good agreement. They also agree with the "entanglement boundary" between the NNN-Haldane phase and the dimerized phase. The firstorder phase transition from Haldane to the dimerized phase is well located by all methods. The most reliable phase boundary between Haldane and NNN-Haldane phases is obtained by the kink in the energy of the central bond. Since on the one hand, the kink in the $\epsilon_{\text {mid }}$ for large $J_{3}$ has vanishing finite-size effect [see Fig. 6(b)] and on the other hand the kink for small $J_{3}$ appears only in large systems, we determined the boundary of the phases with the energy of the central bonds of the largest cluster to which we have access $\epsilon_{\text {mid }}(N=150)$. We cannot
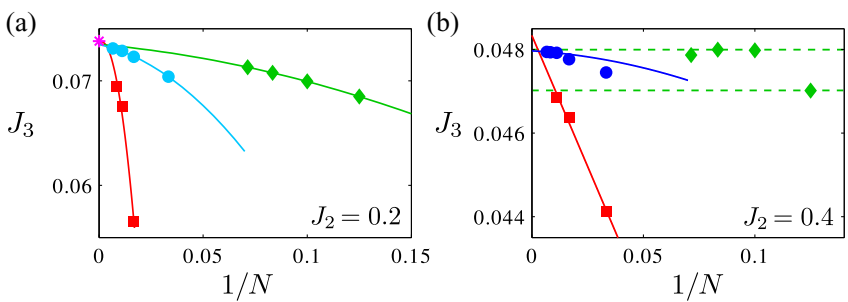

(c)

(d)
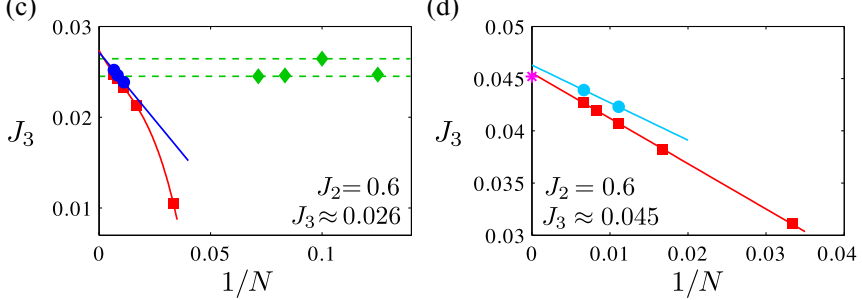

FIG. 14. Comparison of finite-size scaling for (a) $J_{2}=0.2$, (b) $J_{2}=0.4$, (c) $J_{2}=0.6$ and $J_{3} \simeq 0.026$, (d) $J_{2}=0.6$ and $J_{3} \simeq 0.045$. Finite-size results for: entanglement spectra (red squares), the Berry phase (green diamonds), kink in the energy of the central bond $\epsilon_{\text {mid }}$ (blue circles), minimum in the second derivative of $\epsilon_{\text {mid }}$ (cyan circles). Results from finite-size scaling of the dimerization parameter (magenta stars). All fitting curves are polynomial in $1 / N$. Dashed green lines shows the interval between the smallest and the biggest values deduced from the Berry phase.

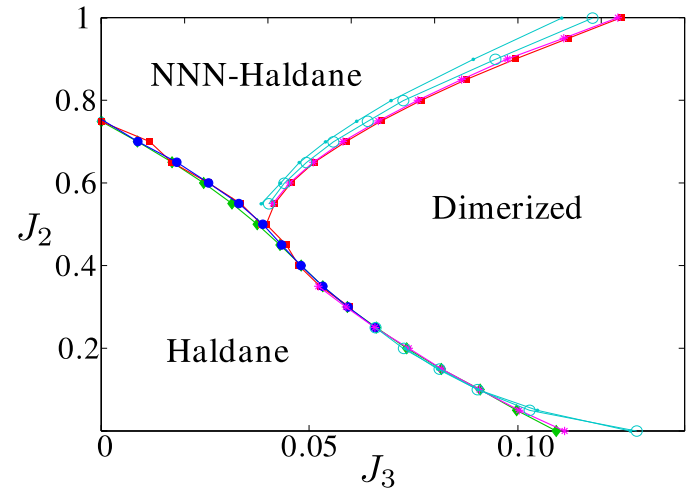

FIG. 15. Comparative phase diagram obtained by dimerization parameter (magenta stars), kink in the energy of the central bond $\epsilon_{\text {mid }}$ (blue circles), $\epsilon_{\text {mid }}(N=150)$, and $\epsilon_{\text {mid }}(N=90)$ (cyan open circles and dots), entanglement spectra (red squares), and Berry phase (green diamonds).

see a kink for $J_{3}=0$ and to locate the phase transition on the $J_{2}$ axis we have used the minimum in the second derivative of the energy with respect to $J_{2}$. The error in the "entanglement boundary" is due to the abrupt change of the degeneracy from two in the Haldane phase to one in the NNN-Haldane or three in the dimerized. The finite-size results of the Berry phase for $N=12$ agree with the "energy boundary" except for $0.5 \leqslant J_{2} \leqslant 0.6$. The finite-size extrapolation of the Berry phase is close to the "dimerization boundary."

\section{SOLITONS AT THE FIRST-ORDER TRANSITION BETWEEN HALDANE AND DIMERIZED PHASES}

We have studied numerically the soliton formation around the first-order phase transition between the Haldane and dimerized phases. In Fig. 16, we show results for the lowestlying $S_{\text {tot }}^{z}=1$ states of a $N=121$ site chain for $J_{2}=0.3$ and different values of $J_{3}$. The most relevant quantities are (i) the local magnetization $\left\langle S_{j}^{z}\right\rangle$ that reveals edge states or solitons; (ii) the spin-spin correlation between nearest neighbors $\left\langle S_{j}^{z} S_{j+1}^{z}\right\rangle$ that reflects the presence of dimerization; and (iii) the expectation value of the three-site interaction $\left\langle\left(\mathbf{S}_{i-1} \cdot \mathbf{S}_{i}\right)\left(\mathbf{S}_{i} \cdot \mathbf{S}_{i+1}\right)+\right.$ H.c. $\rangle$, an indicator of the Haldane phase - it is large and positive in the Haldane phase since spinspin correlations on adjacent bonds are (almost) equal and negative, it is very small and positive in the dimerized phase close to the transition, it vanishes when the state is exactly dimerized, and it is negative everywhere else in dimerized phase.

Our main results can be summarized as follows. Deep inside the Haldane phase there are spin-1/2 edge states as seen from the local magnetization of Fig. 16(a). The small dimerization and the large expectation value of the three-body interaction all along the chain confirm that the entire chain is in the Haldane phase. Around the phase transition, two phases coexist: the dimerized state is favoured close to the edges, while the central part of a chain remains in the Haldane phase [Figs. 16(e) and 16(f)]. The two humps of the local magnetization curve [Fig. 16(d)] show that free spins have moved away from the boundaries and form a pair of spin-1/2 solitons that separates the Haldane and dimerized domains. Deep inside the dimerized phase, the two spins $-1 / 2$ recombine 

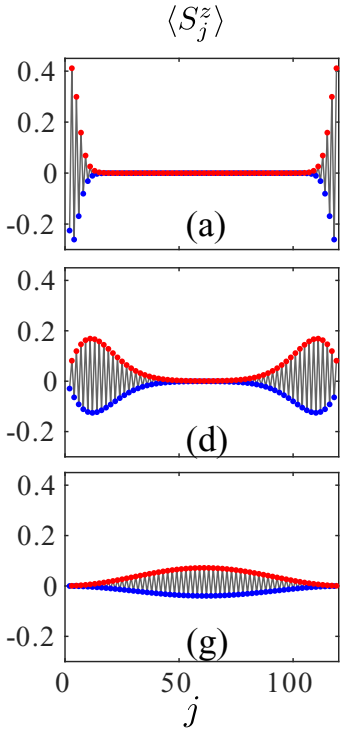
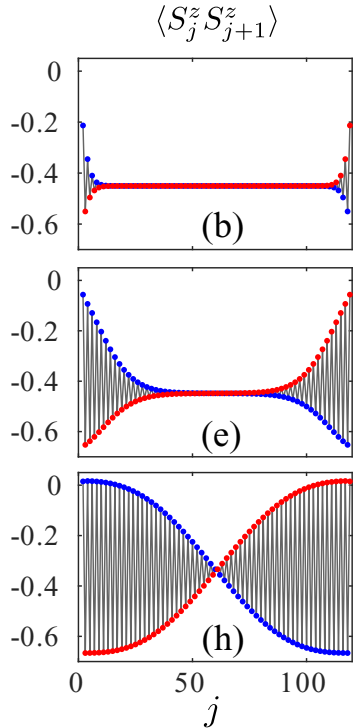
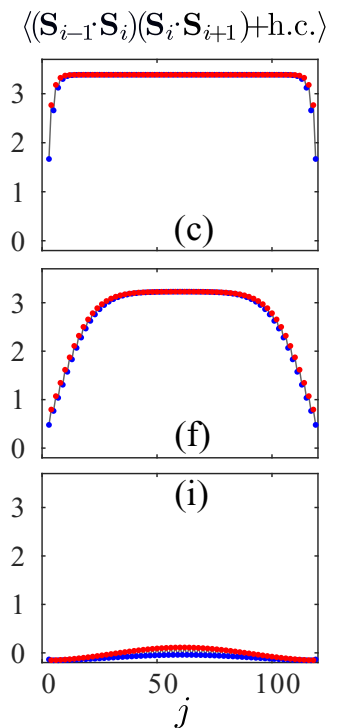
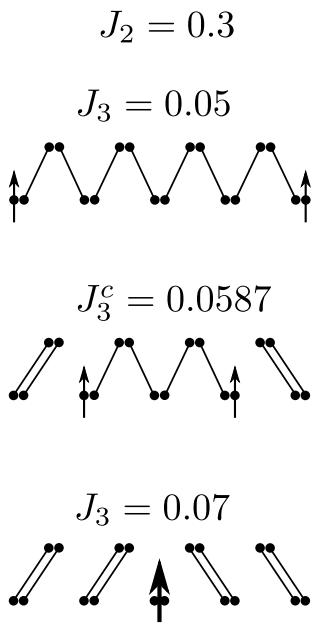

FIG. 16. Spin solitons in chains with $N=121$ at $J_{2}=0.3$ and $S_{\text {tot }}^{z}=1$ below (a)-(c), above (g)-(i), and on the critical line (d)-(f). (Left) On-site magnetization. It reveals (a) spin- $1 / 2$ edge states, (d) spin- $1 / 2$ solitons inside the chain, and (g) spin-1 soliton. (Middle) Spin-spin correlations. They provide evidence of a small dimerization all along the chain in the Haldane phase (b), of a large dimerization at the edges and of its fast decrease in the middle, when the two phases coexist (e), and of a large dimerization all along the chain except in the narrow window in the middle, where the spin-1 soliton is located (h). (Right) Expectation value of the three-body term. It is large and positive all along the chain in he Haldane phase (c), it is small at the dimerized edges but remains large in a domain of Haldane phase in the middle of a chain (f), and it almost vanishes in the dimerized phase (i). The sketches on the right show the VBS picture of solitons in different phases. Thin and thick arrows indicates spin-1/2 and spin-1 solitons. For clarity, each even (odd) data point corresponds to a blue (red) symbol.

into a delocalized spin $S=1$, and two dimerized domains with different orientations occupy half of the chain each [Figs. 16(g)-16(i)]. The transition between two dimerization domains with different dimer orientations can also be deduced from the crossing of the lines formed by red and blue points in the spin-spin correlation [see Fig. 16(h)].

The soliton picture remains true for higher values of the total spin. Figure 17 provides an example of solitons in a chain with $N=120$ and $S_{\text {tot }}^{z}=2$. As in the previous case, one can clearly distinguish spin-1/2 edge states in Fig. 17(a). On top of it, a slight increase of the on-site magnetization occurs in the middle of the chain, indicating the appearance of a spin-1 bond in the bulk. The Haldane phase is then perturbed. This is reflected in the suppressed three-body term measured in the middle of the chain [Fig. 17(c)]. The formation of the dimerized phase starts at the edges, but also in the bulk when approaching the phase transition. Different dimerization domains are separated by domains of Haldane phase, each carrying a total spin 1. Numerically, three dimerization domains are clearly seen with the spin-spin correlations in Fig. 17(e), while the two
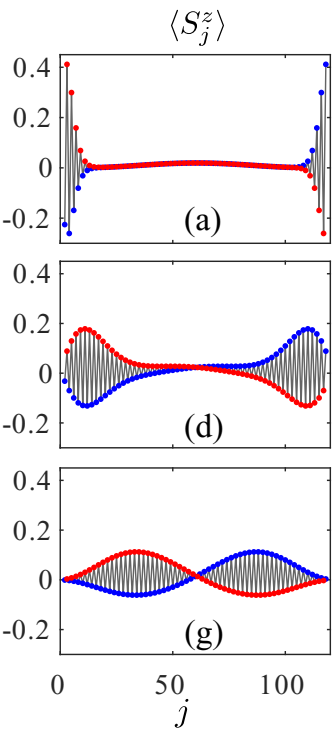

$\left\langle S_{j}^{z} S_{j+1}^{z}\right\rangle$
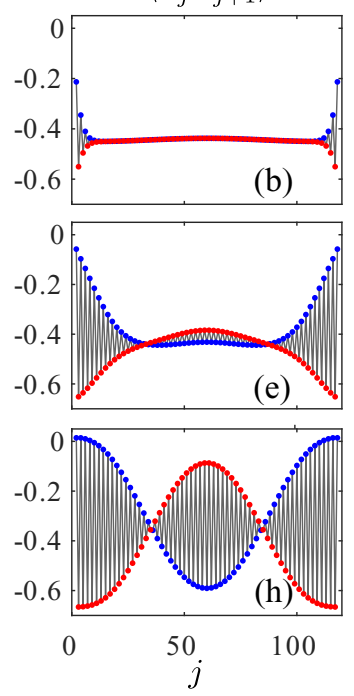

$\left\langle\left(\mathbf{S}_{i-1} \mathbf{S}_{i}\right)\left(\mathbf{S}_{i} \cdot \mathbf{S}_{i+1}\right)+\right.$ h.c. $\rangle$
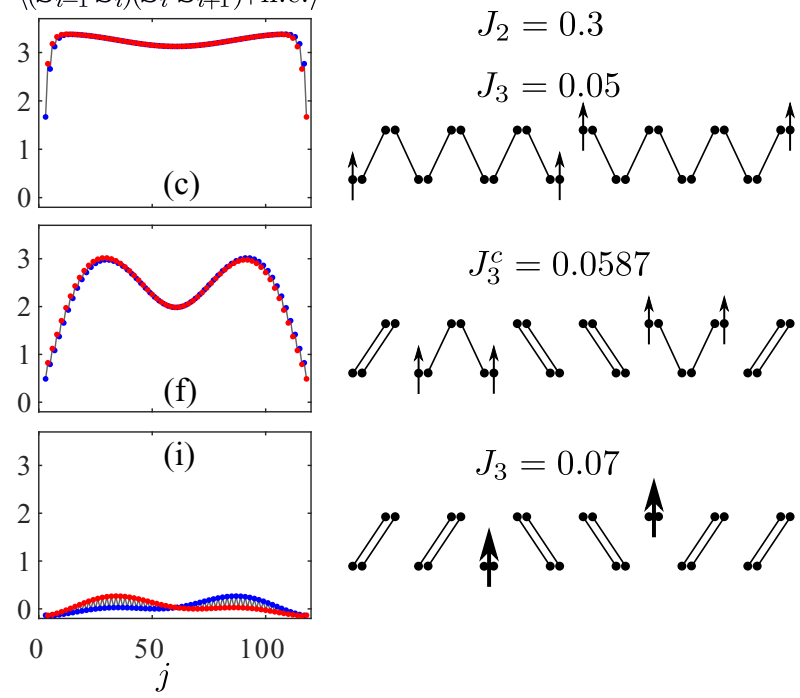

FIG. 17. Same as Fig. 16 for $N=120$ at $J_{2}=0.3$ and $S_{\text {tot }}^{z}=2$ (see main text for the details). 
maxima in the three-body term correspond to two Haldane domains. Inside the dimerized phase, the Haldane domains are suppressed, the dimerization is essentially different from zero everywhere along the chain except at two points symmetric with respect to the middle of the chain. The domain walls are located at the points with vanishing dimerization. The four spins $1 / 2$ recombine into a pair of spins 1 , each of them delocalized along half the chain. The lines formed by red and blue points in the spin-spin correlation intersect twice in Figs. 17(e) and 17(c), implying that the orientation of the dimers is different in neighboring domains.

\section{ISING TRANSITION}

Previously, it was shown that the transition between the NNN-Haldane and dimerized phases is in the Ising universality class at a generic point on the transition line [13]. In this section, we numerically check that the universality class remains the same at the two edges of the transition line: at the triple point where three phases touch each other, and in the limit of large $J_{2}$ and $J_{3}$ couplings.

\section{A. Triple point}

There are two possible scenarios for the triple point of Ising critical line: it can be in either in the Ising or in the tricritical Ising universality class. According to conformal field theory, the first scenario is characterized by a scaling dimension $d=1 / 8$ and a central charge $c=1 / 2$, while the second one is characterized by $d=1 / 24$ and $c=7 / 10$.

We have looked at the critical point along a line that is very close to the first-order transition and perpendicular to the Ising critical line. According to conformal field theory, the local dimerization depends on the chain length $N$ and bond index $j$ as $D(j, N)=[N \sin (\pi j / N)]^{-d}$. The values of $J_{2}$ and $J_{3}$, for which the scaling of the mid-chain dimerization $D(N / 2, N)$ is a separatrix is taken as the critical point [Fig. 18(a)]. At the critical point, the fit of $D(j, N)$ is also good [Fig. 18(b)]. The resulting values of $d \approx 0.158$ and 0.155 point rather towards Ising than towards tricritical Ising criticality.

The central charge was extracted at the critical point from the scaling of entanglement entropy with block size in open systems. Following Ref. [31], we defined the reduced entanglement entropy $\tilde{S}_{N}(n)$ as the one with removed Friedel oscillations:

$$
\tilde{S}_{N}(n)=S_{N}(n)-\zeta\left\langle\mathbf{S}_{n} \mathbf{S}_{n+1}\right\rangle
$$

where $\zeta$ is a numerical parameter. Then, according to conformal field theory the reduced entanglement entropy scales with conformal distance $d(n)=\frac{2 N}{\pi} \sin \left(\frac{\pi n}{N}\right)$ according to

$$
\tilde{S}_{N}(n)=\frac{c}{6} \log d(n)+s_{1}+\log g .
$$

Although our numerical result point out to a central charge $c \approx$ 0.6 , that is in between the two expected values, the monotonous decrease and the fact that for $N=150$ and 200 the central charge is below 0.7 suggest that the critical point is in the Ising universality class.
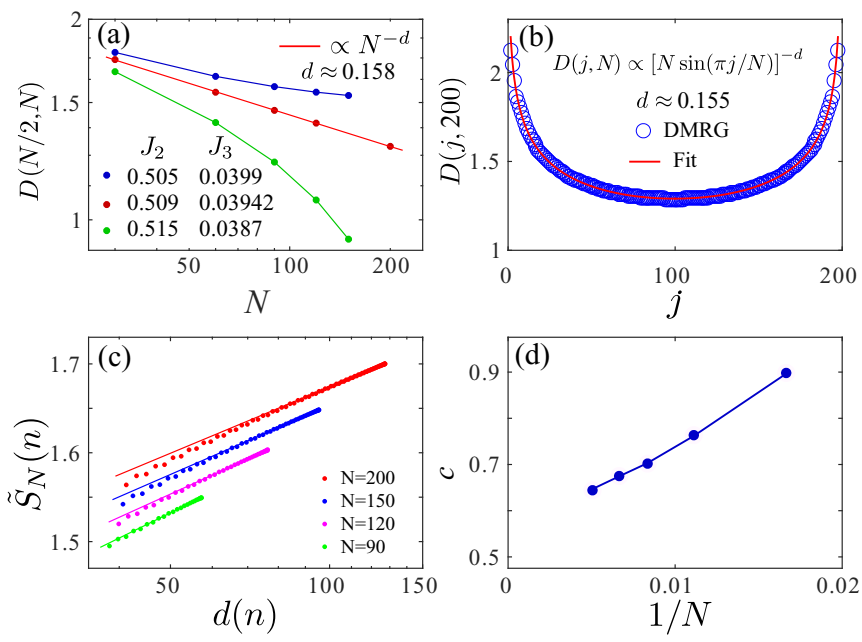

FIG. 18. (a) Log-log plot of the mid-chain dimerization as a function of the number of sites $\mathrm{N}$ for different parameters $J_{2}$ and $J_{3}$ along the line close to the first-order phase transition and perpendicular to the Ising critical line. The linear curve corresponds to the critical point and the slope gives the critical exponent $d \approx 0.158$. (b) Site dependence of $D(j, N)$ at the critical point fitted to $1 /[N \sin (\pi j / N)]^{d}$. This determines the exponent $d \approx 0.155$. (c) Scaling of the entanglement entropy of open chains after removing the Friedel oscillations with conformal distance $d(n)$. (d) Central charge extracted from the entanglement entropy of open chains as a function of $1 / N$.

\section{B. $J_{2}-J_{3}$ model}

We have studied the limit of large $J_{2}$ and $J_{3}$ couplings by setting the nearest neighbor interaction to zero: $J_{1}=0$. As above, we locate the critical point by looking for the separatrix in the scaling of the mid-chain dimerization $D(N / 2, N)$ with the chain length $N$. The slope gives a critical exponent $d \approx 0.126$, in excellent agreement with the Ising one [see Fig. 19(a)]. We also looked at the local dimerization $D(j, N)$ as a function of the bond position $j$. Although the dimerization remains large close to the boundary, one can clearly see that some edge effects appear in the absence of a $J_{1}$ coupling. A similar picture arises in the Ising chain in a transverse field when a magnetic field is applied to the first and last sites of
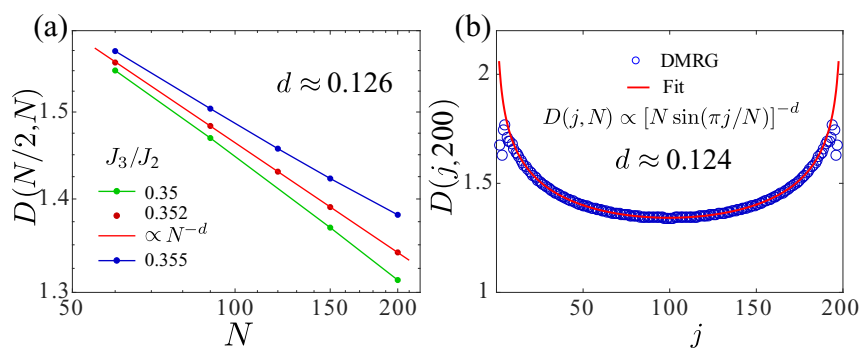

FIG. 19. (a) Log-log plot of the mid-chain dimerization as a function of the number of sites $N$ for $J_{1}=0, J_{2}=1$, and different values of $J_{3}$. The linear curve corresponds to the Ising critical point and the slope gives a critical exponent $d=0.126$, in good agreement with $1 / 8$ for the Ising transition. (b) Site dependence of $D(j, N)$ at the critical point fitted to $1 /[N \sin (\pi j / N)]^{d}$. This leads to an exponent $d=0.124$, again close to the Ising prediction $1 / 8$. 

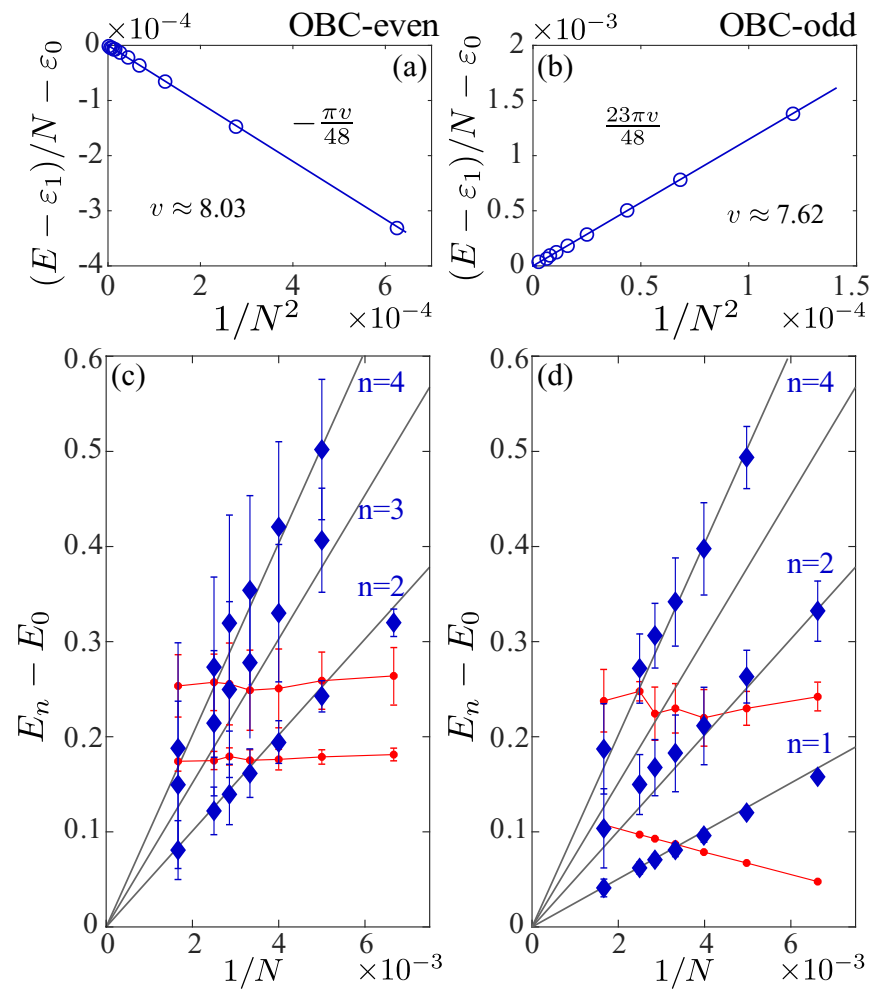

FIG. 20. Ground state and excitation energy as $J_{1}=0, J_{2}=1$, and $J_{3}=0.352$, on the Ising line. (a) and (b) Linear scaling of the ground-state energy per site in an open chain with $1 / N^{2}$ after subtracting the $\varepsilon_{0}$ and $\varepsilon_{1}$ terms for even (a) and odd (b) number of sites. (c) and (d) Energy gap in singlet (blue) and triplet (red) sectors for OBC as a function of $1 / N$ for even and odd number of sites. Each magnetic excitation is twofold degenerate. Grey lines mark the Ising conformal towers of $I$ (c) and of $\epsilon$ (d) with the velocity $v=8.03$ deduced from the finite-size scaling of the ground state energy for even $N$.

the chain with the same orientation if this field is weak as compared to the transverse field. The system renormalizes to fixed boundary conditions at large enough length scales (far enough from the edge) but when the boundary field is weak this corresponding length becomes long. We have thus excluded a few edge points from the fit. The rest of the curve is again in excellent agreement with the Ising prediction $d=1 / 8$ [see Fig. 19(b)].

As predicted by boundary conformal field theory, for the Ising critical point the ground-state energy of an open system with an even number of sites scales as $E=\varepsilon_{0} N+\varepsilon_{1}-$ $\pi v /(48 N)$, where $\varepsilon_{0}$ is a ground-state energy per site, $\varepsilon_{1}$ is a nonuniversal constant, and $v$ is the velocity. For odd $N$ the scaling is of the form $E=\varepsilon_{0} N+\varepsilon_{1}+23 \pi v /(48 N)$. We present the fit of the numerical data in the Figs. 20(a) and 20(b). The extracted values of the velocities $v_{\text {even }} \approx 8.03$ and $v_{\text {odd }} \approx 7.62$ are in reasonable agreement with each other.

As discussed in Ref. [13], if the formation of a dimer is favored on the edge bonds, the chain with even (odd) number of sites $N$ is identified with $\uparrow, \uparrow(\uparrow, \downarrow)$ boundary conditions in the Ising model. Then the conformal tower for even $N$ corresponds to the Ising conformal tower of $I$, while for odd $N$ it corresponds to the Ising conformal tower of $\epsilon$. We have

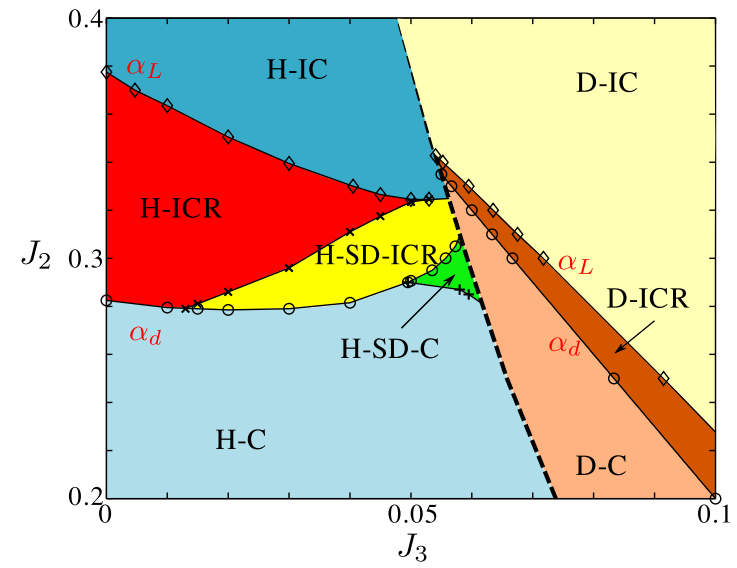

FIG. 21. Enlarged part of phase diagram on Fig. 2 indicating short-range order within the thermodynamic phases. Lifshitz line $\alpha_{L}$ is marked with diamonds and disorder line $\alpha_{d}$ is marked with open circles. Dashed line is a line of the first-order phase transition.

used the velocity $v_{\text {even }}$ deduced from the finite-size scaling of the ground state energy for even $N$ in order to plot the Ising towers of $I$ and of $\epsilon$ in Figs. 20(c) and 20(d) as references.

We have calculated the excitation energy for even and odd numbers of sites in the singlet $S_{\text {tot }}^{z}=0$ and triplet $S_{\text {tot }}^{z}=1$ sectors. The absence of the $J_{1}$ term releases low-lying magnetic excitations that are shown with red lines in Figs. 20(c) and 20(d). Each red line is twofold degenerate, corresponding to the excitation close to the left and to the right edges. The first singlet excitation appears below the triplet one only for $N>300$. By looking at the excitation energy as a function of the number of DMRG iterations, or, more specifically, as a function of the position of the state tensor updated at each iteration, we were able to distinguish bulk excitations from the excitations at the edges, even when they were above the first triplet excitation. Note that by edge excitation we understand a local magnetic excitation of a bond that is located close to the chain boundary.

Since the calculations had to be done for very large systems [in Figs. 20(c) and 20(d)], we present the results for $N$ in the range 150 to 601 ), the convergence of the algorithm is quite slow, implying significant error bars. In systems with nonzero $J_{1}$ coupling, we saw that for an odd number of sites the fourth excitation was more stable in the DMRG sense than the third one. This explains the "missing" third excitation on panel (d): we were not able to converge enough excited states for systems that are so large. To summarize, we have have provided numerical evidence that the phase transition between the NNN-Haldane and dimerized phases is always in the Ising universality class, including at the triple point where the Haldane, NNN-Haldane and dimerized phases touch, and in the limiting case of the $J_{2}-J_{3}$ model.

\section{SHORT-RANGE ORDER}

\section{A. Disorder and Lifshitz lines}

As mentioned in Sec. II, several types of short-range order are present in the Haldane, NNN-Haldane and dimerized phases (see Fig. 21). A detailed description of each phase 
(a)

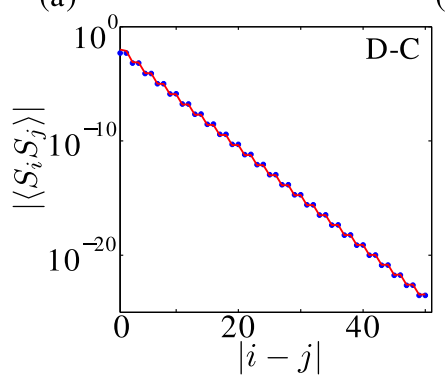

(b)

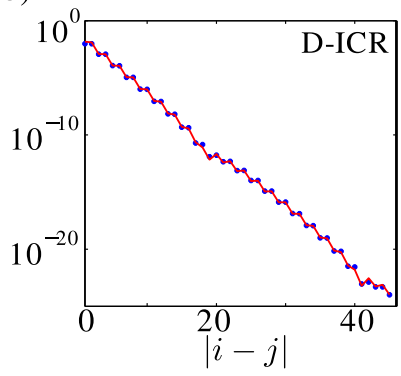

FIG. 22. Spin-spin correlation function $\left\langle S_{i} S_{j}\right\rangle$ for $J_{2}=0.25$ with (a) $J_{3}=0.083$ and (b) $J_{3}=0.084$; the red lines are fit to the data with the dimerized OZ form given by Eq. (4) with (a) $q=\pi$ and (b) $q>\pi$.

has already be given in Sec. II. In this section, we describe the numerical results that led to this phase diagram in more detail.

The most important result is that, by tuning either the nextnearest-neighbor or the three-body interaction, short-range incommensurate order can be induced beyond the so-called disorder and Lifshitz lines. Disorder points were first discussed by Stephenson in models of classical statistical mechanics [3234]. On one side of a disorder point, the correlation function decays in a commensurate way, while on the other side it decays in an incommensurate way. The disorder point is said to be of the first kind if the wave number in the incommensurate phase depends on the temperature, and of the second kind if it does not [33]. In the present case, we have only found disorder points of the first kind.

By contrast, at a Lifshitz transition, the spin-spin correlation function becomes incommensurate in momentum space, each peak being replaced by two symmetric peaks in the structure factor $S F(q)$ defined in Eq. (5). By keeping track of real-space and momentum-space correlations, we found that disorder and Lifshitz lines cross the transition line at $J_{2} \simeq 0.335$ and $J_{2} \simeq$ 0.342 .

\section{B. Dimerized phase}

By fitting the numerical results of the spin-spin correlations with the dimerized OZ form given by Eq. (4), we have extracted
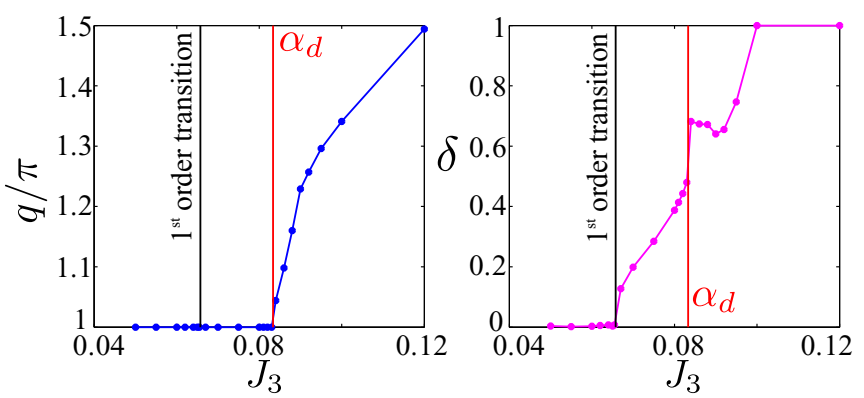

FIG. 23. Wave number $q$ and dimerization $\delta$ deduced from a fit of the spin-spin correlation function with Eq. (4) for $J_{2}=0.25$. The position of the first-order phase transition and the line $\alpha_{d}$ where the ground state is fully dimerized are marked with black and red lines, respectively.

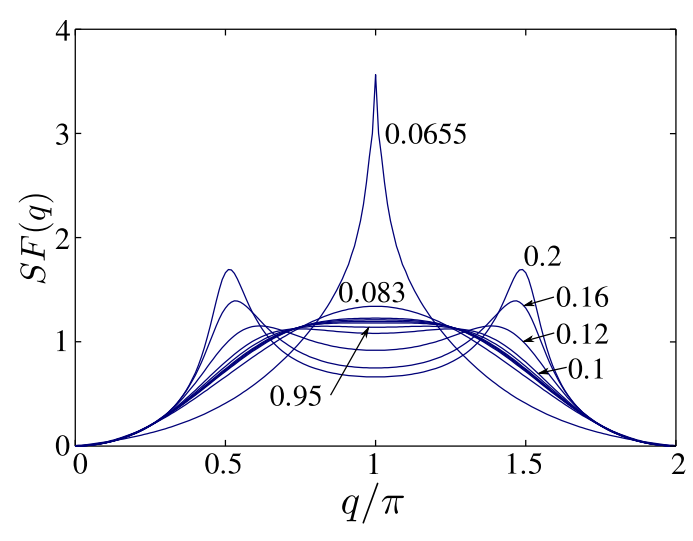

FIG. 24. Structure factor $S F(q)$ for $J_{2}=0.25$ and various values of $J_{3}$. The Lifshitz point is at $J_{3}=0.0915 \pm 0.0005$.

the wave number $q$ and the short-range dimerization parameter $\delta$. Examples of fits for $J_{2}=0.25$ are shown in Fig. 22 .

We have found that, with very high accuracy, the disorder line coincides with the line where the fully dimerized wave function is the exact ground state of the model (see Fig. 23). In order to determine the Lifshitz line, we have looked for the appearance of a two-peak structure in $S F(q)$ given by Eq. (5), where we have restricted the sum to the interval $20<i, j \leqslant$ $N-20$ in order to eliminate edge effects. Some examples of structure factor calculated for fixed $J_{2}=0.25$ are presented in Fig. 24.

The conclusion that emerges from these results is that, to go from the commensurate to the incommensurate part of the dimerized phase, one has to cross first a disorder line, and then a Lifshitz line. These results are very similar to those obtained for the spin-1/2 chain with next-nearest-neighbor interaction, the fully dimerized line of our model being the equivalent of the Majumdar-Ghosh point [1]. At that point, the correlation length vanishes, and it coincides with the disorder point $J_{2}^{d}=1 / 2$, while the Lifshitz point of the spin- $1 / 2$ chain is located at $J_{2}^{L}=0.52036(6)$ [35], well above the disorder point.

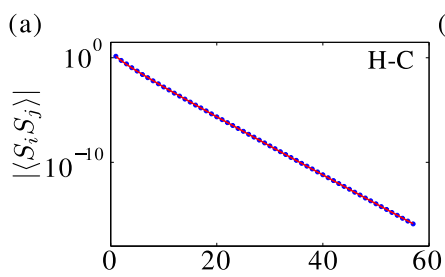

(b)

(c)

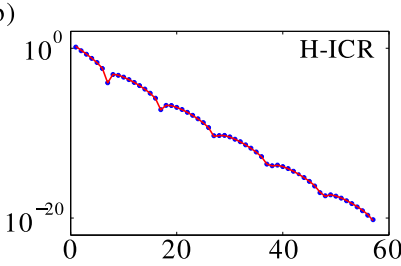

(d)
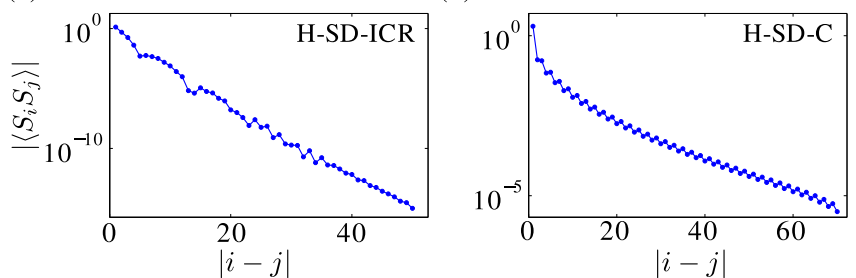

FIG. 25. Spin-spin correlation function $\left\langle S_{i} S_{j}\right\rangle$ for (a) $J_{2}=0.27$, $J_{3}=0$; (b) $J_{2}=0.3, J_{3}=0$; (c) $J_{2}=0.3, J_{3}=0.04$, and (d) $J_{2}=$ $0.3, J_{3}=0.059$. The red line on (a) and (b) is a fit to the data with the $\mathrm{OZ}$ form. 

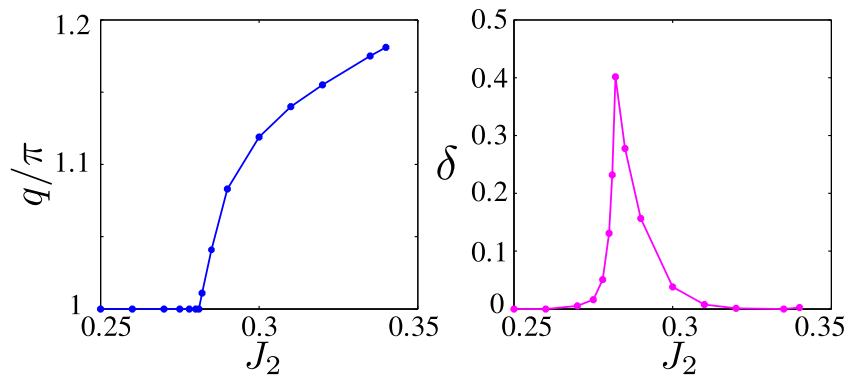

FIG. 26. Wave number $q$ and dimerization $\delta$ deduced from a fit of the spin-spin correlation function with Eq. (4) for $J_{3}=0.03$.

\section{Haldane phase}

Depending on the type of correlation in the Haldane phase we have fitted the numerical data with either nondimerized $\mathrm{OZ}$ or dimerized OZ forms given by Eqs. (3) and (4). Figure 25 provides several examples of spin-spin correlations and some fits.

The wave number $q$ and the short-range dimerization parameter $\delta$ extracted from the fit for fixed $J_{3}=0.03$ are summarized in Fig. 26. Note that there is a finite region where the dimerization is essentially different from zero.

Crossing the transition line at $J_{2} \simeq 0.335$, the disorder line is separated from the transition line in Haldane phase by a thin tail of commensurate phase with short-order dimerization (H-SD-C). The Lifshitz line in the Haldane phase is obtained in the same way as in the dimerized phase. Close to the crossing point $J_{2} \simeq 0.342$, the Lifshitz line is very close to the boundary of the H-SD-ICR phase, making the H-ICR phase vanishingly small in this region.

\section{CONCLUSION}

Combining field theory arguments with DMRG (and occasionally exact diagonalizations), we have shown that the dimerization transitions of the spin-1 Heisenberg model with next-nearest neighbor and three-site interaction can be precisely located and fully characterized. In particular, the transition between the Haldane phase and the dimerized phase is in the $\mathrm{SU}(2)_{2}$ WZW universality class for small $J_{2}$, and it becomes first order at an end point also in the $\mathrm{SU}(2)_{2}$ WZW universality class. Along the first-order line between these phases, the solitons between Haldane and dimerized phases carry a spin- $1 / 2$, in qualitative agreement with the fact that along the $\mathrm{SU}(2)_{2}$ line, there are low-lying magnetic excitations. By contrast, the transition between the next-nearest neighbor Haldane phase and the dimerized phase is in the Ising universality class. Along this transition line, the spin-gap remains open, and the low-lying excitations are all in the singlet sector.

To fully characterize the transitions, DMRG with open boundary conditions turned out to be extremely useful. This is due to the fact that the conformal tower of a critical model with open boundary conditions is often just the tower of a single primary field. By contrast, the conformal tower of a critical model with periodic boundary conditions is in general the superposition of different towers. We think that a systematic use of these ideas might turn out to be useful in other one-dimensional quantum systems.

In addition, we have shown that short-range correlations can be commensurate or incommensurate, with several disorder and Lifshitz lines, leading to a remarkably rich phase diagram. Interestingly, several of these phases occur for relatively small, hence physically realistic values of the couplings $J_{2}$ and $J_{3}$. So it is our hope that the present investigation will encourage experimentalists to try and check some aspects of this phase diagram.

\section{ACKNOWLEDGMENTS}

We are indebted to Andreas Läuchli and Andrey Nevidomskyy for insightful discussions and advises. The first evidence of a partially first-order transition between the Haldane phase and the dimerized phase has been obtained by Clément Bazin during his Master thesis. This work has been supported by the Swiss National Science Foundation and by NSERC of Canada, Discovery Grant 04033-2016 (IA) and the Canadian Institute for Advanced Research (IA).
[1] C. K. Majumdar and D. K. Ghosh, J. Math. Phys. 10, 1388 (1969).

[2] K. Okamoto and K. Nomura, Phys. Lett. A 169, 433 (1992).

[3] L. A. Takhtajan, Phys. Lett. A 87, 479 (1982).

[4] H. M. Babujian, Nucl. Phys. B 215, 317 (1983).

[5] I. Affleck, Nucl. Phys. B 265, 409 (1986).

[6] I. Affleck, Phys. Rev. Lett. 56, 746 (1986).

[7] I. Affleck and F. D. M. Haldane, Phys. Rev. B 36, 5291 (1987).

[8] A. Kolezhuk, R. Roth, and U. Schollwöck, Phys. Rev. Lett. 77, 5142 (1996).

[9] A. K. Kolezhuk and U. Schollwöck, Phys. Rev. B 65, 100401 (2002).

[10] F. Michaud, F. Vernay, S. R. Manmana, and F. Mila, Phys. Rev. Lett. 108, 127202 (2012).

[11] F. Michaud, S. R. Manmana, and F. Mila, Phys. Rev. B 87, 140404 (2013).
[12] J. H. Pixley, A. Shashi, and A. H. Nevidomskyy, Phys. Rev. B 90, 214426 (2014)

[13] N. Chepiga, I. Affleck, and F. Mila, Phys. Rev. B 93, 241108(R) (2016).

[14] N. Chepiga, I. Affleck, and F. Mila, Phys. Rev. B 94, 136401 (2016).

[15] F. D. M. Haldane, Phys. Lett. A 93, 464 (1983).

[16] T. Kennedy, J. Phys.: Condens. Matter 2, 5737 (1990).

[17] F. Pollmann, A. M. Turner, E. Berg, and M. Oshikawa, Phys. Rev. B 81, 064439 (2010).

[18] A. Kolezhuk, R. Roth, and U. Schollwöck, Phys. Rev. B 55, 8928 (1997).

[19] S. R. White, Phys. Rev. Lett. 69, 2863 (1992).

[20] U. Schollwöck, Rev. Mod. Phys. 77, 259 (2005).

[21] Z.-Y. Wang, S. C. Furuya, M. Nakamura, and R. Komakura, Phys. Rev. B 88, 224419 (2013). 
[22] S. Östlund and S. Rommer, Phys. Rev. Lett. 75, 3537 (1995).

[23] U. Schollwöck, Ann. Phys. 326, 96 (2011).

[24] M. V. Berry, Proc. R. Soc. London A 392, 45 (1984).

[25] T. Hirano, H. Katsura, and Y. Hatsugai, Phys. Rev. B 77, 094431 (2008).

[26] P. Di Francesco, P. Mathieu, and D. Sénéchal, Conformal Field Theory, Graduate Texts in Contemporary Physics (Springer, New York, 1997).

[27] Z.-C. Gu and X.-G. Wen, Phys. Rev. B 80, 155131 (2009).

[28] H. Li and F. D. M. Haldane, Phys. Rev. Lett. 101, 010504 (2008).
[29] M. Levin and X.-G. Wen, Phys. Rev. Lett. 96, 110405 (2006).

[30] A. Kitaev and J. Preskill, Phys. Rev. Lett. 96, 110404 (2006).

[31] S. Capponi, P. Lecheminant, and M. Moliner, Phys. Rev. B 88, 075132 (2013).

[32] J. Stephenson, Can. J. Phys. 47, 2621 (1969).

[33] J. Stephenson, Phys. Rev. B 1, 4405 (1970).

[34] J. Stephenson and D. D. Betts, Phys. Rev. B 2, 2702 (1970).

[35] R. Bursill et al., J. Phys.: Condens. Matter 7, 8605 (1995). 